\title{
Characterization of colloidal structures during intestinal lipolysis using Small-Angle Neutron Scattering
}

Oljora Rezhdo ${ }^{\mathrm{a}}$, Selena Di Maio ${ }^{\mathrm{b}}$, Peisi Le ${ }^{\mathrm{c}}$, Kenneth C. Littrell ${ }^{\mathrm{d}}$, Rebecca L. Carrier ${ }^{\mathrm{a}}$, Sow-Hsin Chen ${ }^{\mathrm{c}^{*}}$.

* Both authors contributed equally

a. Department of Chemical Engineering, Northeastern University, 360 Huntington Ave., Boston, MA 02151, USA

b. Department of Medicine, University of California San Francisco, 405 Parnassus Ave., San Francisco, CA 94143, USA

c. Department of Nuclear Engineering, Massachusetts Institute of Technology, 77 Massachusetts Ave., Cambridge, MA 02139, USA

d. High Flux Isotope Reactor, Oak Ridge National Laboratory, 1 Bethel Valley Rd., Oak Ridge, TN 37831, USA

\section{Oljora Rezhdo}

313 Snell Engineering Center

360 Huntington Avenue

Boston, MA 02115, USA

o.rezhdo@neu.edu

\section{Selena Di Maio}

513 Parnassus Ave, Med Sci Room 1350

San Francisco, CA 94143, USA

selena.dimaio@ucsf.edu

\section{Peisi Le}

77 Massachusetts Avenue, 24-302

Cambridge, MA 02139, USA

lepeisi@mit.edu

\section{Kenneth Charles Littrell}

Oak Ridge National Laboratory, HFIR

1 Bethel Valley Road,

Oak Ridge, TN 37831

littrellkc@ornl.gov 
Corresponding Authors:

Rebecca Lyn Carrier

313 Snell Engineering Center

360 Huntington Avenue

Boston, MA 02115, USA

r.carrier@neu.edu

Tel: +1 617-373-7126

Fax: +1 617-373-2209

\section{Sow-Hsin Chen}

77 Massachusetts Avenue, 24-209A

Cambridge, MA 02139, USA

sowhsin@ mit.edu

Tel: +1 617-253-3810

Fax: +1 617-899-5027 


\begin{abstract}
Hypothesis: Bile micelles are thought to mediate intestinal absorption, in part by providing a phase into which compounds can partition. Solubilizing capacity of bile micelles is enhanced during the digestion of fat rich food. We hypothesized that the intestinal digestion of triglycerides causes an increase in volume of micelles that can be quantitatively monitored over the course of digestion using small-angle neutron scattering (SANS), and that SANS can enable evaluation of the contribution of each of the components present during digestion to the size of micelles.

Experiments: SANS was used to characterize the size and shape of micelles present prior to and during the in vitro simulated intestinal digestion of a model food-associated lipid, triolein.

Findings: Pre-lipolysis mixtures of a bile salt and phospholipid simulating bile concentrations in fed conditions were organized in micelles with an average volume of $40 \mathrm{~nm}^{3}$. During lipolysis, the micelle volume increased 2.5-fold over a 2-hour digestion period due to growth in one direction as a result of insertion of monoglycerides and fatty acids. These efforts represent a basis for quantitative mechanistic understanding of changes in solubilizing capacity of the intestinal milieu upon ingestion of a fat-rich meal.
\end{abstract}

Keywords: Small-angle neutron scattering, digestion, bile micelles, triglyceride, fatty acid, monoglyceride, diglyceride, simulated intestinal fluids, colloidal structures ${ }^{1}$

\footnotetext{
${ }^{1}$ Abbreviations:

SANS - Small-Angle Neutron Scattering

FA - fatty acid

MG - monoglyceride

DG - diglyceride

$\mathrm{TG}$ - triglyceride

GI - gastrointestinal

PC - phosphatidylcholine

PL - phospholipid

BS - bile salt

NaTDC - sodium taurodeoxycholate

$\mathrm{D}_{2} \mathrm{O}$ - deuterium oxide

SLD - scattering length density
} 


\section{Introduction}

Ingested lipids, originating from food or from drug delivery excipients, can have a significant impact on overall absorption, in particular, of lipophilic drug compounds [1-3]. However, these effects are not quantitatively predictable, in part due to incomplete mechanistic understanding of drug-lipid interactions during gastrointestinal (GI) digestion. One method by which lipids can impact drug fate in the GI tract is by enhancing the drug solubility and kinetics of dissolution [3], which is often the rate-limiting step to overall drug absorption. Drug solubility and dissolution are directly dependent on the phases present in the intestinal lumen post lipid ingestion (e.g., oil phase, micellar phase), their respective volume fractions, and drug partition coefficients.

During intestinal digestion, ingested lipids form oil emulsions partially stabilized by bile secretions. Lipase released into the intestine as part of the enzymatic cocktail in pancreatic juice attacks the oil droplets, digesting triglycerides (TG) - a major component of ingested lipids into diglycerides (DG), monoglycerides (MG), and fatty acids (FA) [4]. Due to the hydrophilic nature of lipase, lipid digestion occurs at the oil-water interface. The products of lipolysis, i.e. DG, MG, and FA, are amphiphilic molecules that in turn aid in the emulsification of lipids and form complex colloidal structures, such as micelles and vesicles, in the aqueous lumen upon mixing with the amphiphilic components of bile $[3,5,6]$. This cascade of processes changes the volume fraction of lipophilic (e.g., oil droplets) and amphiphilic (e.g., bile micelles) phases as well as their chemical nature, leading to altered drug dissolution kinetics, partitioning, and ultimately overall absorption. Thus, a quantitative assessment of changes in phase volume during lipolysis is critical in predicting the impact of lipids on these processes. 
A wide range of biophysical methods including polarized microscopy, X-ray diffraction, and small-angle X-ray scattering (SAXS) have been employed to understand structure of colloids in the GI tract, primarily to study the impact of lipolysis on structural changes in the surface of lipid droplets [7-9]. SANS and SAXS have been predominantly used to characterize the structure of bile micelles, as it is effective in analyzing shape and dimensions of structures falling in the size range of micelles, and SANS can provide information on the internal molecular arrangement within the micelles [10]. Hjelm et al. pioneered the characterization of bile micelle structure using SANS [11-14]. They described the micelles as rod-like cylindrical structures with a radius of approximately $28 \AA$. Within the context of the cylindrical shape model, they proposed the radial-shell configuration, with molecules positioned radially from the central axis, to be the most plausible conformation based on neutron contrast analyses and molecular dimension considerations. Based on this configuration, the radius of the cylinder should correspond to the length of the molecule(s) contained in the micelle. In the presence of FA and MG, the cylindrical micelles elongated in length, suggesting similar behavior may be occurring in the intestine during the digestion of lipids [11-14]. An adjustment to the cylindrical configuration has been proposed in more recent studies, where the caps of the cylinder consist of round surfaces [15]. Other studies, however, have concluded that bile micelles are spheroidal/ellipsoidal in shape [1618]. This discrepancy in reported micelle shape between studies has been in part attributed to the relative concentrations of the micellar components, specifically the relative concentrations of bile salts and phospholipids $[19,20]$.

In addition to colloid structure characterization in static systems as described in the examples above, studies of colloid evolution in dynamic systems undergoing in vitro lipolysis have also been reported in the literature. Many of these studies have looked at changes in micelle 
structure as well as formation of crystalline phases at the oil emulsion interface, some of them using synchrotron SAXS to allow detection of structures throughout lipolysis [21-26]. Such studies have been informative in developing a physical conceptual model of the dynamic intestinal milieu. However, a quantitative assessment of volume evolution of each colloidal phase (i.e. oil emulsions, bile micelles) during lipolysis, critical information that can enable quantitative prediction of drug partitioning into these phases and thus drug dissolution kinetics, has not been presented.

The current work complements these previous studies as it provides a quantitative measurement of the impact of lipolysis on the total micellar phase volume. SANS was used to characterize the structure of micelles and total micelle volume evolution during lipid digestion due to SANS's unique ability to employ the contrast variation technique, a powerful tool that exploits changes in scattering intensity of the neutron beam under different degrees of deuteration of chemical components in solution. External contrast variation analysis was performed on micelle-rich simulated intestinal fluids by changing the $\mathrm{D}_{2} \mathrm{O} / \mathrm{H}_{2} \mathrm{O}$ ratio of the aqueous environment, and, after fitting a micelle shape model, it allowed calculation of volume fraction of the micellar phase and micelle concentration in solution. In this study, we tested three different shape models for the bile micelles, specifically the cylinder, round capped cylinder, and spheroid. In addition, we monitored micellar size evolution in real time during lipid digestion. To represent food-associated lipid intake, triolein was used as a model lipid substrate, and was digested in vitro in the presence of micelle-rich simulated intestinal fluids and the enzymatic cocktail extracted from porcine pancreas.

The contribution of each component present in the simulated intestinal milieu during digestion to the structure of bile micelles was also analyzed. Specifically, the biorelevant 
medium containing micelles was mixed with TG, the enzymatic cocktail, and the products of lipolysis: FA, MG, and DG, separately, to allow mechanistic understanding of the factors influencing the structure of micelles during an in vitro lipid digestion. As micelle components are known to partition into oil droplets, the structure and/or amount of bile micelles may be affected by the presence of TG. Products of lipolysis, given their amphiphilic nature, may partition into the micelles and thus influence their size and possibly transform them into vesicles at certain surfactant concentrations and compositions.

This study is the first report of an in vitro lipolysis model coupled with the SANS technique that provides characterization of dynamic bile micelle volume changes during lipid digestion. This information could ultimately contribute to a comprehensive mechanistic understanding and prediction of the influence of ingested lipids on lipophilic compound absorption.

\section{Materials and Methods}

\subsection{Materials}

Trizma ${ }^{\circledR}$ maleate, sodium chloride $(\mathrm{NaCl})$, calcium chloride dihydrate $\left(\mathrm{CaCl}_{2} * 2 \mathrm{H}_{2} \mathrm{O}\right)$, sodium hydroxide $(\mathrm{NaOH})$, sodium taurodeoxycholate (NaTDC or BS, cat. \# T0875), L-alphaphosphatidylcholine from egg yolk (PC), Type XVI-E (cat. \# P3556), glyceryl trioleate or triolein, pancreatin from porcine pancreas (cat. \# L3126), deuterated water $\left(\mathrm{D}_{2} \mathrm{O}\right)$, 4bromophenylboronic acid, oleic acid, 1-oleoyl-rac-glycerol, and 1,3-diolein were purchased from Sigma-Aldrich Co., St. Louis, MO, USA.

\subsection{SANS analysis and data collection}

SANS is a powerful tool for determining size, shape, and internal chemical structure of particles in a liquid medium in the size range of roughly 1-200 nm. Using a collimated, 
monochromatic incident neutron beam, the scattering pattern is collected into an area detector as a 2D image. This isotropically scattered pattern of neutrons can be reduced to a 1D graph displaying the relation between the magnitude of the scattering vector, $Q\left(\AA^{-1}\right)$, and the intensity distribution of the scattered neutrons, $\mathrm{I}\left(\mathrm{cm}^{-1}\right)$. The scattering intensity plot that is generated is scaled to absolute values using a correction factor obtained using a standard sample of known absolute scattering cross-section. The experiments shown in this study were conducted at GPSANS at the High Flux Isotope Reactor (HFIR) in Oak Ridge National Laboratory (ORNL) and at NG-7 in the National Institute of Standards and Technology (NIST) Center for Neutron Research (NCNR). At HFIR geological samples were used as standards for absolute intensity scaling, whereas at NCNR we used pyrex glass as a standard.

To observe a wide Q range, three different instrument configurations were used. Each configuration corresponded to a certain distance of the detector from the sample. For data collected at HFIR, 3 different distances were used, $0.3 \mathrm{~m}$ (at wavelength $4.72 \AA$ ), $7 \mathrm{~m}$ (at wavelength $4.72 \AA$ ), and $18.5 \mathrm{~m}$ (at wavelength $12 \AA$ ). Due to instrument differences, at NCNR slightly different sample-to-detector distances and wavelengths for the three configurations were used to maintain the same $\mathrm{Q}$ range. The configurations, each corresponding to a certain $\mathrm{Q}$ range, were named short, medium, and long sample-to-detector distances, respectively. The short configuration corresponds to $0.1<\mathrm{Q}<1 \AA^{-1}$, medium corresponds to $0.01<\mathrm{Q}<0.1 \AA^{-1}$, and long corresponds to $0.001<\mathrm{Q}<0.01 \AA^{-1}$. The wavelength resolutions of the GP-SANS at HFIR and NG-7 at NCNR were 0.14 and 0.12 , respectively.

The sample cells were made of quartz and had a thickness of $2 \mathrm{~mm}$. Considering that the maximum intensity of the scattering at the lowest accessible Q was less than $1 \%$ of the empty direct beam intensity, using sample cells of this thickness, it was assumed that multiple scattering 
was a low probability event, and thus no correction for multiple scattering was performed. Sample cells were mounted on brass holders whose temperature was maintained at $37^{\circ} \mathrm{C}$. The measurement time was 1.5 hours for each sample at ORNL and 1 hour at NCNR. The reduction of the data was done using the Igor Pro software by WaveMetrics, Inc., following protocols developed by the respective facilities [27].

Subtraction of scattering due to background (e.g., solvent, sample cell) was performed differently for samples containing some percentage of $\mathrm{H}_{2} \mathrm{O}$ in the buffer vs. samples containing only $\mathrm{D}_{2} \mathrm{O}$. Specifically, in the former case, background subtraction was performed using scattering intensity data of buffer samples at the matching percentage of $\mathrm{H}_{2} \mathrm{O}$ as in the micellerich samples. In the latter case, background subtraction was performed using scattering intensity data of an empty sample cell, as the scattering generated by the micelles was much larger than the scattering generated by the solvent. Following background subtraction, reduced data were plotted as absolute intensity I $\left(\mathrm{cm}^{-1}\right)$ versus scattering angle $\mathrm{Q}\left(\AA^{-1}\right)$ and fitted by SasView [28].

The SasView fitting software was set to use dQ data from the reduced data file to account for instrumental smearing and dI data for scattering intensity weighting. Model constraints were the requirement of positive numbers for all shape dimensions, i.e. radii and lengths, and in the case of the capped cylinder model, the cap radius was not allowed to be less than the radius of the cylinder. Model inputs were the scattering length density (SLD) of the buffer (usually set equal to the SLD of $\mathrm{D}_{2} \mathrm{O}$, except for contrast variation experiments where the buffer SLD was a weighted average of the SLD of $\mathrm{D}_{2} \mathrm{O}$ and $\mathrm{H}_{2} \mathrm{O}$ ) and the SLD of the micelle (determined by external contrast variation - see section 3.1). Model fitting parameters were a solvent background intensity term, scale (i.e. volume fraction), and shape dimensions. The Chi-squared test within SasView was used to assess the goodness of each fit. 


\subsection{Preparation of samples}

SANS was used to characterize the structure of micelles in aqueous simulated intestinal fluids. Micelle concentrations employed in this study are based on those found in fed state [1], but modified slightly with respect to model bile components [29]. The fed state simulated intestinal fluids (SIF) were prepared as shown in Table 1 in either $100 \% \mathrm{D}_{2} \mathrm{O}$ or a mixture of $\mathrm{D}_{2} \mathrm{O}$ and $\mathrm{H}_{2} \mathrm{O}$ depending on the purpose of the experiment. $0.2 \mathrm{M} \mathrm{NaOH}$ solution used for titration was also prepared to match the $\mathrm{D}_{2} \mathrm{O}-\mathrm{H}_{2} \mathrm{O}$ content of the sample being titrated or $\mathrm{pH}$ adjusted. The SIF was mixed overnight at $300 \mathrm{rpm}$ prior to use and maintained at $37^{\circ} \mathrm{C}$.

Table 1: Composition of SIF containing bile salt and phospholipid at fed concentrations.

\begin{tabular}{|c|c|}
\hline \multirow{2}{*}{ Buffer } & $100 \mathrm{mM}$ Trizma® maleate, pH 6.5 \\
& $65 \mathrm{mM} \mathrm{NaCl}$ \\
\hline \multirow{2}{*}{ Bile components } & $10 \mathrm{mM} \mathrm{CaCl}_{2} * 2 \mathrm{H}_{2} \mathrm{O}$ \\
\hline & $12 \mathrm{mM} \mathrm{NaTDC}$ \\
\hline
\end{tabular}

In this study, we report three sets of experiments. First, the external contrast variation technique was employed to determine the SLD of micelles, a critical parameter used for colloid shape model fitting leading to evaluation of the total micellar phase volume and concentration of micelles. The evolution of bile micelles was then analyzed dynamically upon introduction of lipid and throughout the lipid digestion process. Samples were collected at different time points during digestion, inhibited using an inhibitor solution, and measured on the SANS instrument. Lastly, static experiments were conducted to evaluate the effect of each of the components present during digestion on the structure of micelles. The experiments, respective procedures, and the samples collected for SANS measurement are described in Table 2. 
Table 2: Summary of experiments performed in this study.

\begin{tabular}{|c|c|c|}
\hline Experiment & Description & Purpose \\
\hline \multirow{4}{*}{1} & \multirow{4}{*}{$\begin{array}{l}\text { Simulated intestinal fluids (SIF) } \\
\text { at fed bile concentrations } \\
\text { (GP-SANS) }\end{array}$} & To analyze the size and concentration of micelles. \\
\hline & & Preparation \\
\hline & & Samples: SIF in $40 \%-100 \% \mathrm{D}_{2} \mathrm{O}$ \\
\hline & & Background: Buffer in $40-100 \% \mathrm{D}_{2} \mathrm{O}$ \\
\hline \multirow{6}{*}{2} & \multirow{6}{*}{$\begin{array}{l}\text { SIF }+ \text { triolein }+ \text { pancreatic juice } \\
\text { (in vitro digestion) } \\
\text { (GP-SANS } n=2 \text { and NG-7 } n= \\
\text { 1) }\end{array}$} & $\begin{array}{l}\text { To monitor the dynamic evolution of micelle size during } \\
\text { in vitro intestinal digestion. }\end{array}$ \\
\hline & & $\begin{array}{l}\text { Preparation } \\
18 \mathrm{~mL} \text { of } 50 \mathrm{mM} \text { triolein }+\mathrm{SIF} \text { in } 100 \% \mathrm{D}_{2} \mathrm{O} \text { was pre-mixed for } \\
30 \mathrm{~min} \text { at } 300 \mathrm{rpm} \text { and } 37^{\circ} \mathrm{C} \text {. }\end{array}$ \\
\hline & & $\begin{array}{l}2 \mathrm{~mL} \text { of freshly prepared extract of pancreatin from porcine } \\
\text { pancreas in buffer (Table } 1 \text { ) in } 100 \% \mathrm{D}_{2} \mathrm{O}[12,30] \text { was added to } \\
\text { lipid mixture at start of digestion. }\end{array}$ \\
\hline & & $\begin{array}{l}\mathrm{pH} \text { was monitored with } \mathrm{pH} \text { meter and titrated manually to } 6.5 \\
\text { as needed during } 2 \mathrm{~h} \text { using } 0.2 \mathrm{M} \mathrm{NaOH} \text { in } 100 \% \mathrm{D}_{2} \mathrm{O} \text {. }\end{array}$ \\
\hline & & $\begin{array}{l}\text { Samples were collected over time and reaction was inhibited } \\
\text { using } 0.2 \mathrm{~g} / \mathrm{mL} \text { bromophenylboronic acid in methanol at 1:9 } \\
\text { volumetric ratio immediately after collection [31]. }\end{array}$ \\
\hline & & $\begin{array}{l}\text { Samples: Pre-digestion lipid mixture and digested solutions } \\
\text { inhibited at } 5 \mathrm{~min}, 30 \mathrm{~min}, 60 \mathrm{~min}, 90 \mathrm{~min}, 120 \mathrm{~min} \text { post } \\
\text { addition of enzymatic cocktail } \\
\text { Background: Empty cell }\end{array}$ \\
\hline \multirow{7}{*}{3} & \multirow{3}{*}{$\begin{array}{l}\text { a. SIF + triolein } \\
\text { (GP-SANS) }\end{array}$} & $\begin{array}{l}\text { To compare micellar size and concentration in the } \\
\text { presence vs. absence of oil, investigating the possibility } \\
\text { of micelles dispersing to coat the surface of oil droplets. }\end{array}$ \\
\hline & & $\begin{array}{l}\text { Preparation } \\
\text { Solutions of varying concentrations of triolein were mixed with } \\
\text { SIF for } 30 \mathrm{~min} \text { at } 300 \mathrm{rpm} \text { and } 37^{\circ} \mathrm{C} \text { prior to placing in sample } \\
\text { cell and measuring on the instrument }\end{array}$ \\
\hline & & $\begin{array}{l}\text { Samples: SIF in } 100 \% \mathrm{D}_{2} \mathrm{O}+\text { triolein at } 50 \mathrm{mM}, 30 \mathrm{mM}, 5 \mathrm{mM} \text {, } \\
1 \mathrm{mM} \text {, and } 0.05 \mathrm{mM} \text { concentrations } \\
\text { Background: Empty cell }\end{array}$ \\
\hline & \multirow{3}{*}{$\begin{array}{l}\text { b. SIF + pancreatic juice } \\
\text { (GP-SANS) }\end{array}$} & $\begin{array}{l}\text { To determine potential interactions between the } \\
\text { enzymatic cocktail and the micelles by analyzing micellar } \\
\text { size and concentration. }\end{array}$ \\
\hline & & $\begin{array}{l}\text { Preparation } \\
9 \mathrm{~mL} \text { of SIF in } 100 \% \mathrm{D}_{2} \mathrm{O} \text { were mixed with } 1 \mathrm{~mL} \text { pancreatin } \\
\text { extract freshly prepared in } 100 \% \mathrm{D}_{2} \mathrm{O} \text { buffer immediately } \\
\text { followed by inhibitor at } 1: 9 \text { volumetric ratio as described in } \\
\text { experiment } 2 \text {. }\end{array}$ \\
\hline & & $\begin{array}{l}\text { Sample: SIF + pancreatin + inhibitor in } 90 \% \mathrm{D}_{2} \mathrm{O} \text { and } 10 \% \\
\text { methanol } \\
\text { Background: Empty cell }\end{array}$ \\
\hline & $\begin{array}{l}\text { c. SIF + diolein } \\
\text { (GP-SANS) }\end{array}$ & $\begin{array}{l}\text { To determine the ability of DG (a product of digestion) to } \\
\text { partition into the micelles by analyzing size of micelles in } \\
\text { the presence vs. absence of DG. }\end{array}$ \\
\hline
\end{tabular}


Preparation

Sample: SIF in $100 \% \mathrm{D}_{2} \mathrm{O}+10 \mathrm{mM}$ 1,3-diolein (mixed for $1 \mathrm{~h}$, $\mathrm{pH}$ adjusted if needed)

Background: Empty cell

To determine the ability of MG (a product of digestion)

to partition into the micelles by analyzing size of micelles

d. $\mathrm{SIF}+$ monoolein

(GP-SANS)

in the presence vs. absence of MG.

Preparation

Sample: SIF in $100 \% \mathrm{D}_{2} \mathrm{O}+5 \mathrm{mM}$ 1-oleoyl-rac-glycerol (mixed

for $1 \mathrm{~h}, \mathrm{pH}$ adjusted if needed)

Background: Empty cell

To determine the ability of FA (a product of digestion) to partition into the micelles by analyzing size of micelles in

e. SIF + oleic acid

(GP-SANS) the presence vs. absence of FA.

Preparation

Sample: SIF in $100 \% \mathrm{D}_{2} \mathrm{O}+10 \mathrm{mM}$ oleic acid (mixed for $1 \mathrm{~h}, \mathrm{pH}$ adjusted as needed)

Background: Empty cell

For experiment 2, in addition to micelle structure analysis during lipolysis, digestion kinetics was also analyzed using $\mathrm{NaOH}$ titration. $\mathrm{NaOH}$ titration is necessary to maintain the solution $\mathrm{pH}$ at 6.5 , given the ability of lipolysis products (i.e. FA) to lower the solution $\mathrm{pH}$ as digestion proceeds, and the amount of $\mathrm{NaOH}$ added for titration acts as an indicator of the amount of ionized FA in solution. However, depending on the pKa of the acid, only a portion of the FA in solution may be ionized, and thus only some of the FA is accounted for by this method. Often end-point titration is also performed by bringing the solution $\mathrm{pH}$ to 9 to ensure that all the FA in solution is ionized [32]. The pKa of oleic acid, the main product of triolein digestion, has been reported to be anywhere between 7.2 and 9.85 [33-35]. We utilized a Corona charged aerosol detector (CAD, ESI Biosciences) in line with high performance liquid chromatography (HPLC, Agilent Technologies) to allow a more complete assessment of lipids in solution over time, as it could quantify the presence of TG in addition to FA. Specifically, lipolysis samples collected at various time points during digestion were diluted 10X in a 50:50 methanol-chloroform solution immediately upon collection, resulting in precipitation of the 
enzymatic cocktail and thus, inhibition of reaction. Samples were then filtered using a $0.2 \mu \mathrm{m}$ nylon syringe filter and measured on the HPLC using a ProntoSIL 120 phenyl column with $5 \mu \mathrm{m}$ bead size, 250 x $4.6 \mathrm{~mm}$ dimensions, following method proposed by Olsson et al. [36].

Scattering data collection for experiments $3 \mathrm{~d}$ and $3 \mathrm{e}$ employed only the medium (5-50 $\mathrm{nm}$ rough particle diameter range detection) and short $(1-5 \mathrm{~nm}$ rough particle diameter range detection) configurations since the samples were visibly clear, indicating the absence of larger particles which are commonly observed using the long instrument configuration.

\section{Results and Discussion}

This study aimed to quantify the evolution of bile micelles during the in vitro digestion of a triglyceride by means of SANS. In addition, after a thorough structural analysis of the simulated endogenous bile micelles using external contrast variation, the contribution of each of the molecular components present during digestion to structural changes in the micelles was investigated.

\subsection{Determination of the geometry of micelles through external contrast variation} (Experiment 1)

The structure and size of large colloids (e.g., vesicles, oil emulsions) in aspirated human intestinal content have been analyzed, for example using cryo-TEM [37, 38]. In vitro simulated intestinal contents, generally prepared by mixing a bile salt with a phospholipid to mimic bile components, have allowed detailed structural analysis of micelles at concentrations representative of both fasted and fed conditions. SANS and contrast variation have been used to determine SLD, aggregation numbers, and concentration of model bile micelles in solution with various components in the literature [10, 39]. However, the specific simulated intestinal fluids that we have employed in this study containing NaTDC as a model bile salt and egg yolk 
lecithin, at concentrations of $12 \mathrm{mM}$ and $4 \mathrm{mM}$, respectively, have not been previously structurally analyzed. The primary purpose of this experiment was to obtain a micellar SLD ( $\rho$, unit: $\AA^{-2}$ ), as this is a critical input parameter for scattering data fitting, allowing quantification of the concentration (number/fluid volume) of micelles in solution. Knowledge of micellar concentration provides a quantitative means of assessing potential depletion of the micellar phase due to, for example, micellar components partitioning into oil droplets. In addition, micellar concentration allows calculation of the total micellar phase volume once volume of a single micelle is determined (as described below). Total micellar phase volume is an important parameter in determining compound partition coefficients in micelle-water systems. It is currently not clear how the relative percentage of a given compound associated with micelles vs. in aqueous media changes during lipolysis, and knowledge of total micellar volume will allow us to determine if partitioning is governed simply by a larger volume of micelles, or if the changing chemical environment within micelles during lipolysis significantly impacts partitioning.

While the SLD can often be estimated using known chemical formulas of the molecular components and their relative ratios within the micelle by using tabulated neutron scattering lengths [40], this estimation is not possible for the micellar system used in this study. Specifically, the molecular formula of egg yolk PC is not available because it consists of a mixture of lecithins of varying carbon chain lengths. In addition, while the molar ratio of BS and $\mathrm{PC}$ in solution is $3: 1$, the molar ratio within the micelles may not be the same considering that a fraction of these molecules exist free in solution. The intensity scattering plots at varying degrees of solvent deuteration are shown in Figure 1. 


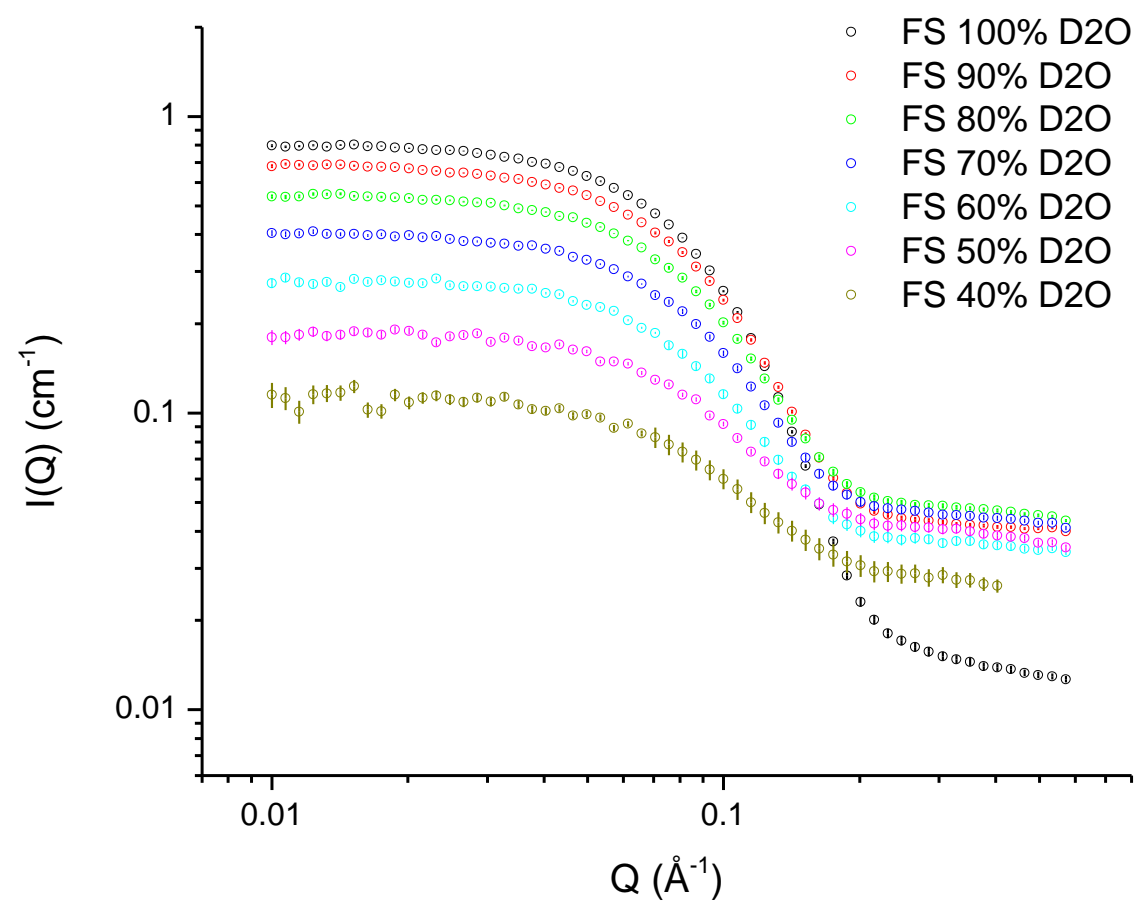

Figure 1: Absolute intensity scattering plots for samples of bile micelles containing bile salt and lecithin at fed state (FS) concentrations using varying ratios of $\mathrm{D}_{2} \mathrm{O} / \mathrm{H}_{2} \mathrm{O}$.

The scattering intensity plots plateau at the low and high $Q$ values indicating that all the structural information of the micelles is captured within the measured Q range. The decrease in the slope of the curve with the decrease in $\mathrm{D}_{2} \mathrm{O}$ content suggests that the SLD of the colloidal particles becomes more and more similar to the SLD of the solvent, i.e. there is less contrast between the micelles and the solvent as expected for decreasing amounts of $\mathrm{D}_{2} \mathrm{O}$. Scattering plot data were analyzed as described by Lin et al. [10] to obtain the SLD of micelles (Figure 2). 


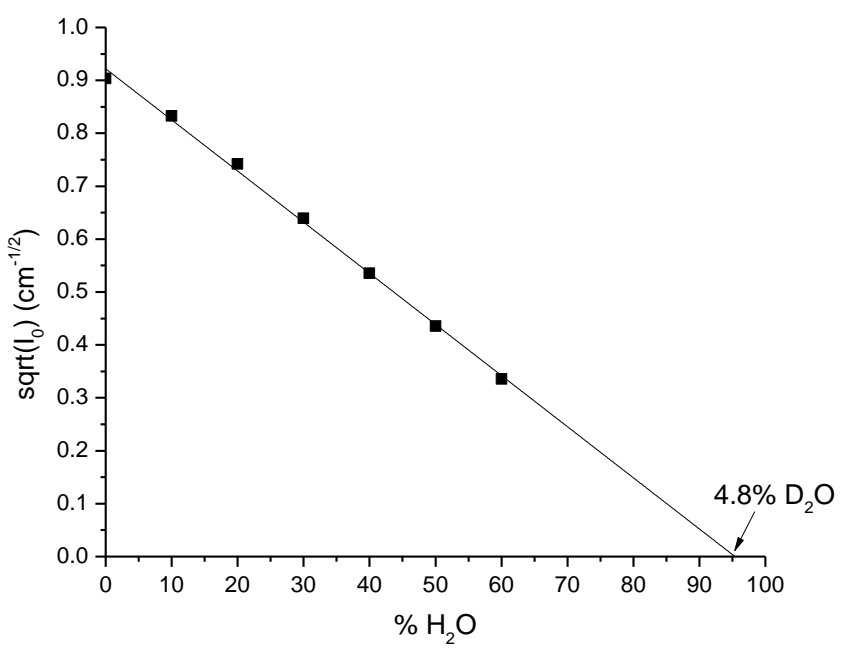

Figure 2: Plots of the square root of scattering intensity of bile micelles at $Q=0 \AA^{-1}$ as a function of $\mathrm{H}_{2} \mathrm{O}$ content in solvent. The $y=0$ point corresponds to a $95.2 \% \mathrm{H}_{2} \mathrm{O}$ content, representing the contrast matching condition for the micelles.

The intercept with the $\mathrm{x}$-axis represents the point at which the contrast of micelles matches the contrast of the solvent. The SLD can be determined as a weighted average of the SLD of $\mathrm{D}_{2} \mathrm{O}\left(\mathrm{SLD}_{\mathrm{D} 2 \mathrm{O}}=6.39 \mathrm{E}-06 \AA^{-2}\right)$ and the SLD of $\mathrm{H}_{2} \mathrm{O}\left(\mathrm{SLD}_{\mathrm{H} 2 \mathrm{O}}=-5.61 \mathrm{E}-07 \AA^{-2}\right)$ at that matching point. The bile micelles are matched at $4.8 \% \mathrm{D}_{2} \mathrm{O}\left(\mathrm{SLD}_{\text {micelles }}=-2.29 \mathrm{E}-07 \AA^{-2}\right)$.

The simulated fed state bile micelle scattering data were fit to three different models, the cylindrical rod-like model [41], the round capped cylinder [42, 43], and the spheroid model [44], considering their use in the literature for such micellar systems [10-18]. Data obtained from samples prepared at $100 \% \mathrm{D}_{2} \mathrm{O}$ were used for model fitting, as they provide the greatest neutron contrast. All models generated close fits to the scattering intensity data with a slight mismatch at $\mathrm{Q} \sim 0.2 \AA$ (Figure 3A for the spheroid, and Figure 1A and 1B in Supplementary Materials for the cylinder and capped cylinder, respectively). The cylinder and capped cylinder models generated similar dimensions, with the cap radius being significantly larger than the cylinder radius, thus resembling a simple cylinder with flat ends. The addition of polydispersity following a Schulz distribution model [45] as a fitting parameter for all dimensions allowed improved goodness of 
fit at the $\mathrm{Q} \sim 0.2 \AA$ mismatch for the cylinder (Figure 1C in Supplementary Materials) and spheroid models (Figure 3B). A singularity error and calculation crash with the SasView software occurred when incorporating polydispersity as a fitting variable with the capped cylinder model. The inclusion of polydispersity for the cylinder model only impacted the cylinder radius, and similarly, the inclusion of polydispersity for the prolate spheroid only impacted the minor radius.
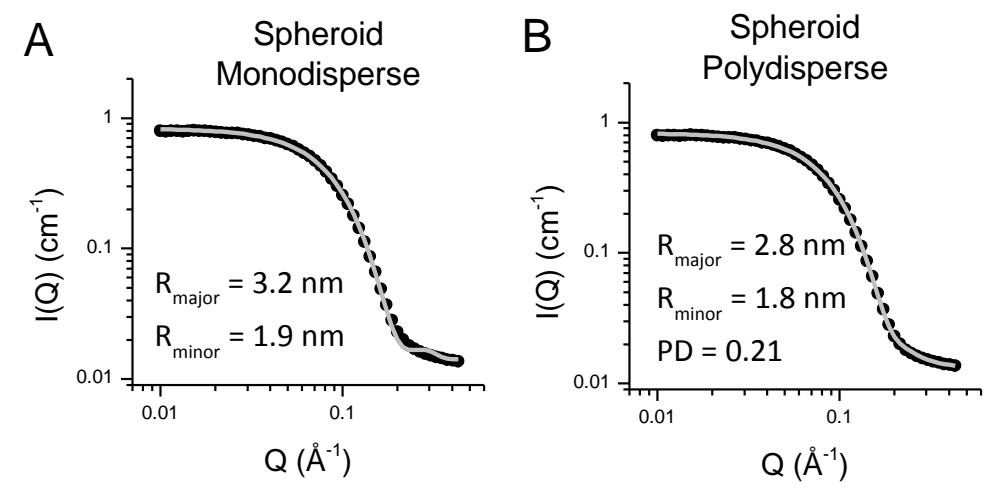

Figure 3: Fits of the simulated fed state bile micelles using the spheroid model using a monodisperse (A) and polydisperse (B) system. The black dots represent the intensity scattering data and the solid gray lines are the SasView [28] fitted curves. Error bars for the intensity scattering data are smaller than the symbol size.

The fitted intensity curves were similar and close to the raw data despite the shape model chosen, thus supporting the applicability of either shape to the micelles studied here. The dimensions are comparable to models of bile micelles reported in the literature $[16,17]$. While the fitting results do not help elucidate the actual shape of these simulated fed state micelles, it is important to note that the total micellar volume in solution is similar among all monodisperse and polydisperse models (Table 3). Additionally, all shape models generated similar micellar volumes and concentrations in the monodisperse and polydisperse groups.

Table 3: Volumetric analysis and comparison of the three shape models employed with and without polydispersity

\begin{tabular}{|c|c|c|c|c|}
\hline Model & $\begin{array}{c}\text { Micellar } \\
\text { Volume }\left(\mathrm{nm}^{\mathbf{3}}\right)\end{array}$ & $\begin{array}{c}\text { Scale }=\text { Total Micellar Volume } \\
\text { Fraction }(\mathbf{m L} / \mathbf{m L} \text { solution) }\end{array}$ & $\begin{array}{c}\text { Conc of micelles } \\
(\# / m L \text { solution) }\end{array}$ & Polydispersity \\
\hline Cylinder & 47 & 0.003998 & $8.60 \times 10+16$ & Monodisperse \\
\hline
\end{tabular}




\begin{tabular}{|c|c|c|c|c|}
\hline & 41 & 0.004097 & $1.00 \times 10+17$ & Polydisperse \\
\hline \multirow{2}{*}{$\begin{array}{c}\text { Capped } \\
\text { cylinder }\end{array}$} & 47 & 0.003936 & $8.31 \times 10+16$ & Monodisperse \\
\cline { 2 - 5 } & N/A & N/A & N/A & Polydisperse \\
\hline \multirow{2}{*}{ Spheroid } & 48 & 0.003851 & $7.97 \times 10+16$ & Monodisperse \\
\cline { 2 - 5 } & 38 & 0.004003 & $1.06 \times 10+17$ & Polydisperse \\
\hline
\end{tabular}

The micellar volume for the two polydisperse fits (i.e. cylinder and spheroid) was calculated by taking into account the polydispersity distribution in the dimensions as described in reference [45]. The scale or volume fraction is a fitting model output defined as the ratio of total micelle volume to the solution volume. The concentration of micelles is then determined as the ratio of scale to the micellar volume. Using these results, we approximated the micellar volume, total micellar volume fraction, and micellar concentration to be $40 \mathrm{~nm}^{3}, 4 \mu \mathrm{L} / \mathrm{mL}$ solution, and 1.03 X 10+17 micelles/mL, respectively.

The lack of knowledge on number, arrangement, or packing of molecules within the micelles made it difficult to elucidate the actual micelle shape. Specifically, despite the known bulk concentrations of NaTDC and egg yolk PC, the unknown critical micellar concentrations of components in this system and existence of polydispersity in micelle size and volume would introduce uncertainties in the determination of an aggregating molecular unit and thus aggregation number. Molecular arrangement and packing are also elusive. The shape of BS molecules is not similar to a conventional surfactant with a hydrophilic head and a hydrophobic tail, but is rather planar with the steroidal nucleus forming the hydrophobic side and the hydroxyl groups and ionic head group forming the hydrophilic side [46, 47]. This suggests that the molecules are likely not tightly packed, and that there are empty spaces within the micelle. In addition, the presence of high electrolyte concentrations in micellar solutions has been shown to generate highly hydrated micelles [48, 49], further contributing to loose packing. Thus, the presence of NaTDC and high electrolyte concentration in our experiments likely contribute to 
spacing between NaTDC and PC molecules within the micelles in this study, introducing complications in assessing molecular packing within the micelles. Studies employing the internal contrast variation technique may help elucidate the shape and internal micellar structure.

For the purposes of this study, it is likely that the volume of the micellar phase is a more relevant factor in understanding the kinetics of compound partitioning into micellar systems than the shape, particularly assuming compound partitioning into micelles is fast relative to partitioning into other colloidal structures in the intestine (e.g., oil emulsions) and is not limited by the interfacial barrier [50], which is dependent on the interfacial surface area and hence a direct function of the micellar shape. Specifically, fast partitioning is governed by the following equation,

$$
K_{\text {micelles } / \text { water }}=\frac{C_{D, \text { micelles }}}{C_{D, \text { water }}}=\frac{n_{D, \text { micelles }} V_{\text {micelles }}}{n_{D, \text { water }} V_{\text {water }}}
$$

where $K_{\text {micelles/water }}$ is the micelle-water partition coefficient, $C_{D, \text { micelles }}$ and $n_{D, \text { micelles }}$ are the concentration and number of moles of drug within the micellar phase, respectively, $C_{D, \text { water }}$ and $n_{D, \text { water }}$ are the concentration and the number of moles of drug in the buffer phase, respectively, $V_{\text {micelles }}$ is the total volume of the micellar phase, and $V_{\text {water }}$ is the total volume of the buffer phase. During lipolysis, the total micellar volume may change due to the formation of new amphiphilic molecules such as fatty acids and monoglycerides. Assuming the partition coefficient remains constant as digestion proceeds, knowledge of dynamic changes in micellar volume provides a means for real-time quantification of the amount of drug partitioned in the micellar phase. In light of such consideration and in the interest of brevity, we chose to pursue the spheroid model (with polydispersity) in fitting the rest of the scattering data presented in this study. 
To provide an indication of experimental variability, we report profiles and fitting results (Figure 4) as well as associated model output parameters (Table 4) of micellar solutions prepared at different times in $100 \% \mathrm{D}_{2} \mathrm{O}$. It is clear that the micelle solutions prepared at different times produce consistently similar micellar sizes and concentrations. In summary, the overall micellar volume fraction is $4.4 \pm 0.7 \mu \mathrm{L} / \mathrm{mL}$ solution and the micelle concentration $9.3 \mathrm{X} 10+16 \pm 1.8 \mathrm{X}$ 10+16 micelles/mL. This average micelle concentration was used to calculate total volume of micellar phase during lipolysis (section 3.2 below).

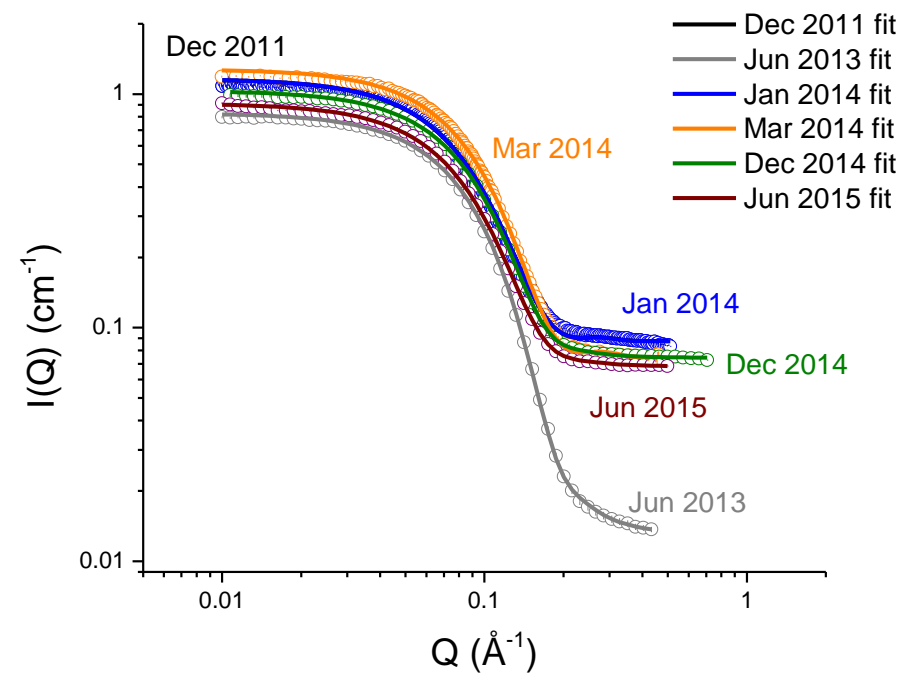

Figure 4: Scattering intensity plots of 6 sets of bile micelle solutions and their respective spheroid shape model fits. Error bars are smaller than symbol size. The scattering intensity profile of the Dec 2011 sample (black) is hidden under the Jan 2014, Dec 2014, and Jun 2015 curves.

Table 4: Spheroid shape model outputs of the scattering intensity plots of independent preparations of micelle solutions

\begin{tabular}{|c|c|c|c|c|c|c|}
\hline $\begin{array}{c}\text { Micelle } \\
\text { solution } \\
\text { preparation } \\
\text { date }\end{array}$ & $\begin{array}{c}\text { Solvent } \\
\text { Background } \\
\left(\mathrm{cm}^{-1}\right)\end{array}$ & $\begin{array}{c}\text { Major } \\
\text { radius }(\mathrm{nm})\end{array}$ & $\begin{array}{c}\text { Minor } \\
\text { radius }(\mathrm{nm})\end{array}$ & $\begin{array}{l}\text { Polydispersity } \\
\text { in minor } \\
\text { radius }\end{array}$ & $\begin{array}{c}\text { Scale } \\
\text { (mL/mL } \\
\text { solution) }\end{array}$ & $\begin{array}{c}\text { Micelle } \\
\text { concentration } \\
(\# / \mathrm{mL})\end{array}$ \\
\hline Dec 2011 & 0.0872 & 3.5 & 1.9 & 0 & 0.00444 & $8.02 \times 10+16$ \\
\hline Jun $2013^{*}$ & 0.0130 & 2.8 & 1.8 & 0.21 & 0.00400 & $1.03 \times 10+17$ \\
\hline Jan 2014 & 0.0871 & 3.5 & 1.9 & 0 & 0.00444 & $8.04 \times 10+16$ \\
\hline Mar 2014 & 0.0743 & 3.2 & 1.9 & 0 & 0.00570 & $1.18 \times 10+17$ \\
\hline Dec 2014 & 0.0744 & 2.9 & 1.8 & 0.20 & 0.00444 & $1.03 \times 10+17$ \\
\hline Jun 2015 & 0.0689 & 3.2 & 1.9 & 0.16 & 0.00351 & $7.02 \times 10+16$ \\
\hline
\end{tabular}


* Same results as those reported in Figure 3 and Table 3

\subsection{Effect of in vitro lipid digestion on size of bile micelles (Experiment 2)}

Previous SANS analysis of bile micelles during lipid digestion has been performed only in static systems where micelles composed of bile salts and phospholipids were mixed with varying concentrations of $\mathrm{MG}$ and $\mathrm{FA}$ in an effort to mimic the products of lipid digestion at varying time points during lipolysis $[12,51]$. A dynamic system in which colloidal structures were analyzed during a digestion process was utilized in this study to provide a more realistic representation of intestinal contents during the digestion of lipids. Figure 5 presents the intensity scattering plots obtained from one of the three sets of digestion experiments, which all produced similar data.

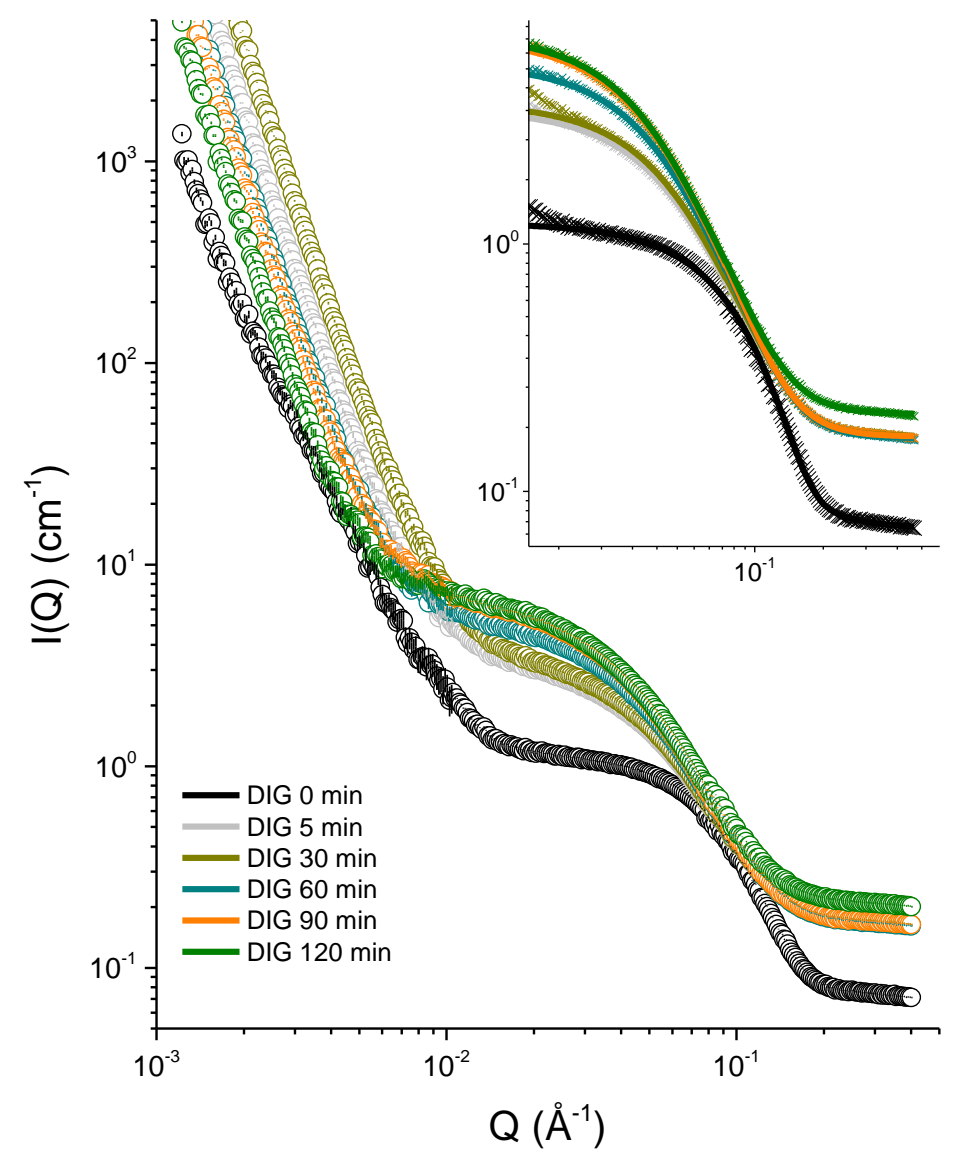

Figure 5: Absolute intensity scattering plots obtained during one of three digestion experiments in which in vitro lipolysis of $50 \mathrm{mM}$ triolein mixed with the fed state micelles was studied. The 'DIG 0 min' sample represents the mixture of oil with the simulated intestinal fluids containing micelles prior to the addition of the enzymatic cocktail. Fits of the $Q>0.01$ 
$\AA^{-1}$ region are shown in the inset graph to avoid cluttering. Notation for the scattering raw data in the inset graph is changed from $Q$ to $\times$ for easier visualization of fitted graphs. Error bars are shown as vertical lines in front of the circles. Most are smaller than the symbol size.

The scattering data in the medium and high $\mathrm{Q}$ region $\left(0.01-0.5 \AA^{-1}\right)$ indicate the presence of bile micelles in Figure 5. The low $\mathrm{Q}\left(\mathrm{Q}<0.01 \AA^{-1}\right)$ region indicates the presence of larger particles, such as oil droplets and protein structures present in the enzymatic cocktail. The absence of a plateau in the low $\mathrm{Q}$ region (below $0.002 \AA^{-1}$ ) provides a hindrance for size characterization of the large particles. Additionally, no useful information could be obtained from the slope of the low $Q$ curve since the intensity, $I(Q)$, is due to a combined contribution of scattering from oil droplets and protein structures. Other attempts were made to obtain oil droplet size information using a Brookhaven Dynamic Light Scattering instrument and a N4 Plus Beckman Coulter particle size analyzer. Despite the presence of surfactants (such as bile salts and phospholipids) in solution, oil droplets form a crude emulsion that is unstable under nonmixing conditions. This fact generated difficulties in obtaining consistent droplet size information using these instruments that require relatively lengthy measurement times (1 minute and 6 minutes, respectively). Further work is currently being conducted in our lab to characterize oil droplet size in real time during in vitro intestinal lipolysis.

It is also noted that the lack of scattering at around $0.007-0.01 \AA^{-1} \mathrm{Q}$ (roughly $100 \mathrm{~nm}$ diameter) indicates that likely no vesicles were formed during the lipolysis of triolein in the presence of fed state micelles, or that their scattering contribution is negligible compared to that of the oil droplets. This result agrees with observations from the literature. Studies have shown that lipids mixed with bile components at fed concentrations are associated with lower aqueous turbidity compared to lipids mixed with bile at fasting concentrations. These results have been attributed to absence of vesicles during fed state lipolysis and co-existence of vesicles with micelles and lipid emulsions during fasted state lipolysis [12]. Indeed, in vitro lipolysis studies 
using cryo-TEM have shown the presence of vesicles when lipids are digested using fasted state concentrations of bile salts [21,23], but vesicles are missing when these lipids are digested using fed state bile concentrations [23].

It is observed that the background scattering (generated by the buffer) for the lipid mixture prior to lipolysis (DIG 0 min sample) at $\mathrm{Q}>0.1 \AA^{-1}$ is noticeably less than the background scattering for the inhibited digestion samples. This is consistent in all three repetitions of the experiment (data not shown). While at first it was believed that the abrupt increase in the background scattering intensity was due to the presence of methanol in the inhibited samples and/or pancreatin extract components, results from experiment $3 \mathrm{~b}$ (see section 3.3 and Figure 10) indicate that these two factors do not impact the background scattering intensity. Hence, it is likely that, as PC undergoes rapid hydrolysis into lyso-PC and FA upon addition of the enzymatic cocktail [52], a fraction of these products, and in particular short and medium chain FA, may partition out of the micelles into the aqueous environment, thus increasing background scattering. Products of triolein digestion are unlikely to partition into the aqueous phase due to the relatively high hydrophobicity of the long chain (C18 FA).

It is important to note that instrument measurement times of the digestion samples were lengthy. Care was taken to perform SANS measurements using the medium configuration $(0.008$ $\AA^{-1}<\mathrm{Q}<0.02 \AA^{-1}$ ) first, such that samples sat unmixed (at $37^{\circ} \mathrm{C}$ ) for at most 10 min prior to collection of scattering data pertaining to micelles. However, samples sat for 1.5-2 hours and 3-8 hours prior to measurements in the short $\left(\mathrm{Q}>0.1 \AA^{-1}\right)$ and long configurations $\left(\mathrm{Q}<0.01 \AA^{-1}\right)$, depending on the order of sample analysis in the instrument. Oil droplet coalescence and enzyme degradation may occur during this time period, which would in turn impact the scattering intensity profile of lipolysis samples in the low $\mathrm{Q}$ region. 
The scattering intensity contribution of the low $\mathrm{Q}$ structures $\left(\mathrm{Q}<0.005 \AA^{-1}\right)$ was fit using an exponential term and was subtracted from the original scattering intensity data to obtain only the scattering intensity profile of micelles. The resultant micellar intensity profile was fit to the chosen shape model, and a volume fraction of the micellar phase was determined using the micelle concentration obtained in section 3.1. The fitting analysis of the three data sets reveals that mixed micelles grow progressively by lengthening along the major axis as digestion proceeds (Figure 6). As a result, the volume of micelles is increased 2.49 fold after 120 minutes of digestion. Model bile micelle volume increase has been shown to occur in the presence of increasing amounts of fatty acids and monoglycerides $[12,51]$, suggesting that these products of digestion have an effect on the size evolution of micelles during lipolysis, as supported by our analysis of a dynamic digestion system. Polydispersity with respect to $\mathrm{R}_{\mathrm{a}}$ remained at 0 , whereas polydispersity with respect to $R_{b}$ increased with each collection time point, from 0.23 at 0 min to 0.46 at $120 \mathrm{~min}$. 


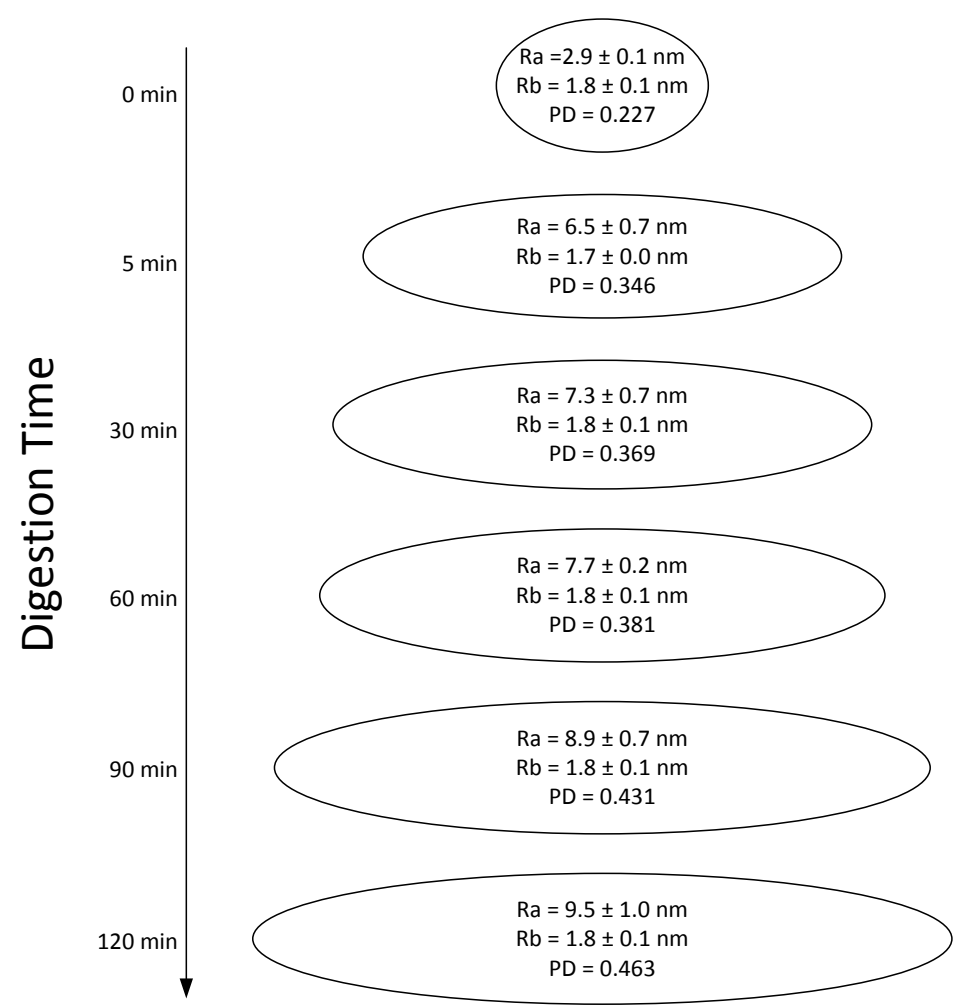

Figure 6: Size and shape of endogenous micelles evolving as a function of the in vitro lipolysis time. The error represents the standard deviation of data from three digestion experiments. PD refers to polydispersity with respect to $R_{b}$. The value has been determined as a ratio of the standard deviation to the mean of the radius.

The volume of micelles during digestion was also calculated from the fitting results and plotted as shown in Figure 7A. The fitting results for the cylinder model are also included for completeness and confirm that the micellar volume is independent of the micellar shape chosen for the fits. Total micellar phase volume (shown in dark cyan in Figure 7A) was also quantified using the concentration of micelles calculated in section 3.1 and the assumption that the number of micelles during digestion remains unchanged. The rate of TG depletion, and thus oil volume change, during lipolysis measured by $\mathrm{CAD}$ is shown for completeness in Figure 2 in the Supplementary Materials section. The kinetics of micellar volume change during digestion is characterized by fast volume expansion at the beginning of lipolysis and slower expansion after 30 minutes. Interestingly, this behavior is similar to the kinetics of FA production during digestion (Figure 7B). Total FA content was quantified via CAD whereas ionized FA content 
was quantified via direct titration at $\mathrm{pH}$ 6.5. It is observed that at the current experimental conditions, specifically, $\mathrm{pH}$, solution ionic strength, and temperature, roughly $50 \%$ of the total oleic acid is ionized and $50 \%$ is protonated. The similarity between the micelle volume and the FA concentration profiles suggests that the change in micellar volume during lipolysis might be directly related to FA production kinetics. Assuming the change in micellar volume due to lipolysis directly impacts compound partitioning in the fed intestine, these results suggest that kinetics of lipolysis could be directly and quantitatively related to the extent of partitioning in this phase during lipolysis. Exploring the relationship between phase volume and compound partitioning is critical in enabling quantitative mechanistic prediction of oral compound behavior in the presence of lipids in the GI tract. Several studies have been reported in the literature where an increase in fatty acid and/or monoglyceride concentration in simulated intestinal fluids causes an increase in oral drug solubility $[53,54]$. This study provides direct evidence of a volume increase of the micellar phase due to the products of lipolysis, that likely contributes, at least in part, to enhancing compound solubility in the micelle-rich aqueous intestinal fluids during digestion. 

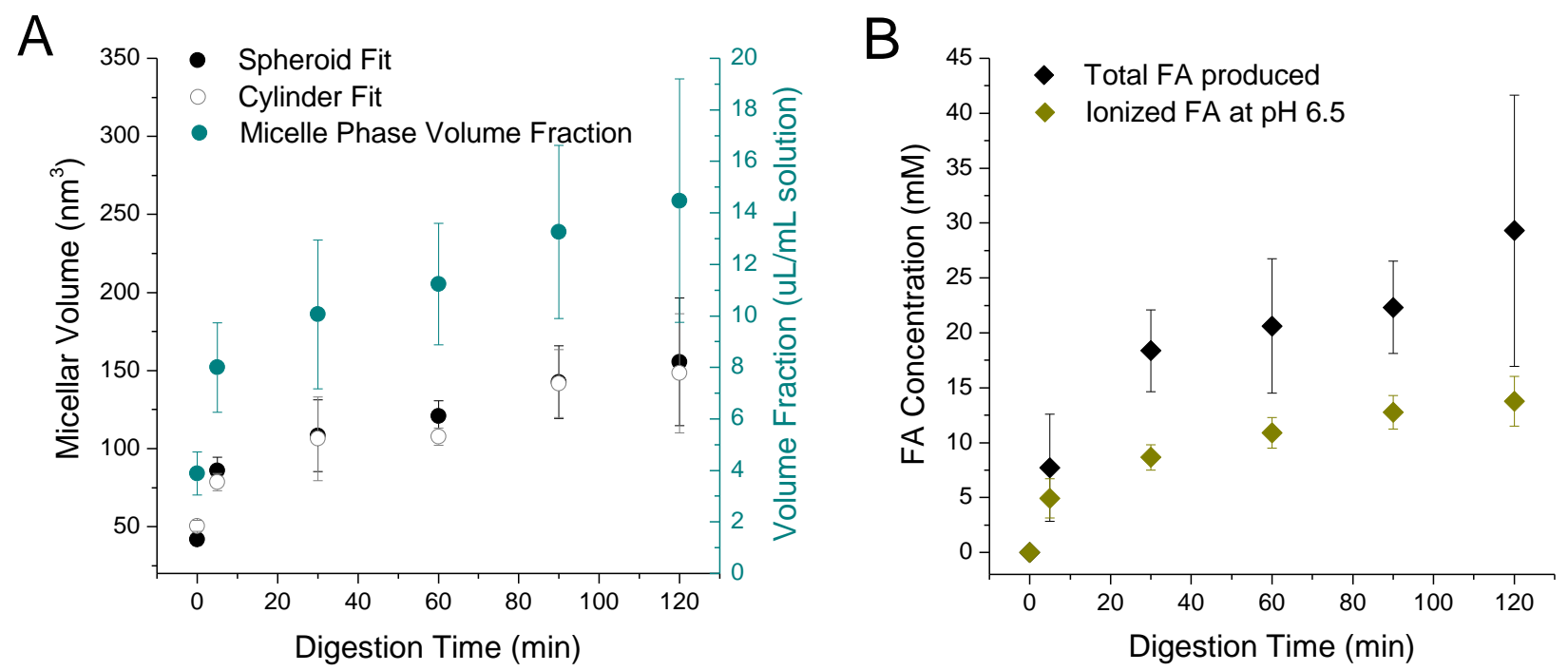

Figure 7: A) Evolution of the volume of micelles during intestinal lipolysis using the spheroid and cylinder models as well as the volume fraction of the total micellar phase. Data represent mean + standard deviation $(n=3)$, B) Kinetics of ionized vs. total FA production during lipolysis of triolein $(n=3)$.

\subsection{Effect of components of oil digestion on simulated bile micelle dimensions (Experiment}

3)

The effect of each of the components present during digestion experiments (triglyceride, enzymatic cocktail, diglyceride, monoglyceride, and fatty acid) on the volume of micelles was examined separately. Here we use the spheroid model to fit and help visualize the effect of these components on the size of micelles. When scattering intensity was present in the low $\mathrm{Q}$ range $(\mathrm{Q}$ $<0.02 \AA^{-1}$ ), the same analysis technique as that applied to the scattering data of digested-andinhibited samples was employed. Specifically, an exponential term was fit to the low Q region of the curve, and this fit was subtracted from the original scattering curve. The resulting curve represented the micelle contribution to the scattering intensity plot and was fit to the shape model.

Triglyceride (Experiment 3a) - The effect of oil droplets on the size and amount of bile micelles has not been studied to date. It is commonly accepted that bile micelles help emulsify lipid droplets by insertion of bile salts at the oil-water interface [46]. This phenomenon could 
result in a depletion of the number of micelles present in solution when lipids are introduced. In addition, in the case of mixed micelles consisting of more than one amphiphilic species, different species may exhibit different affinity for partitioning into the lipid droplets, altering the molecular composition and potentially the size of the remaining micelles.

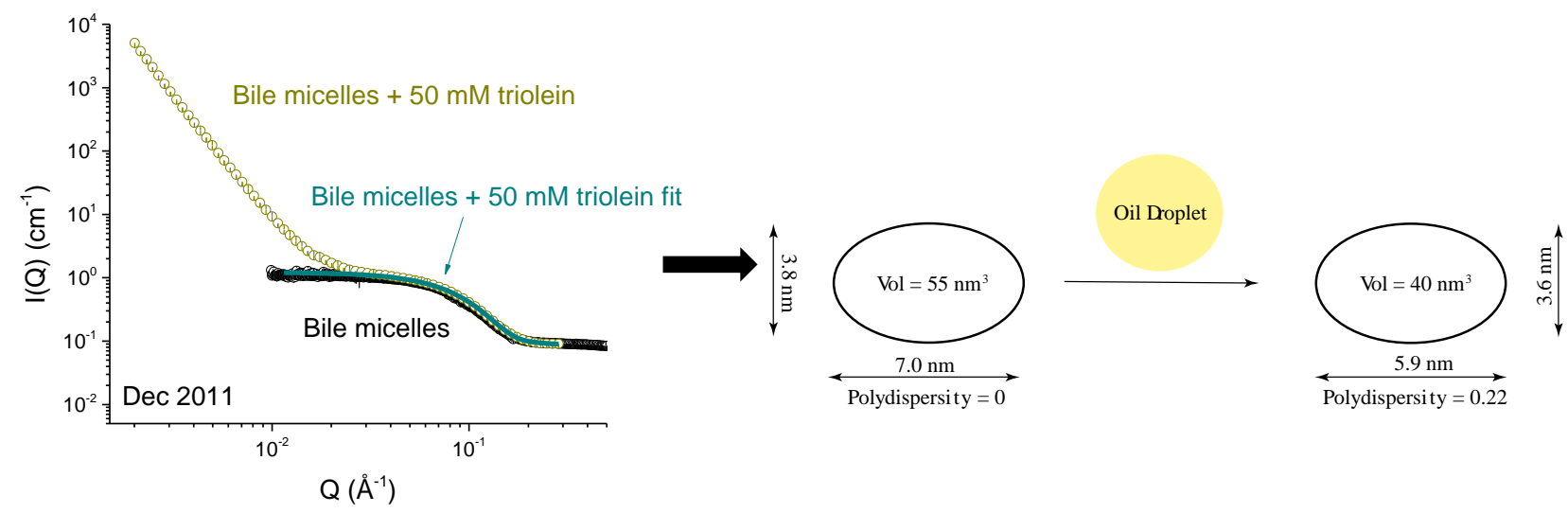

Figure 8: Scattering intensity plot comparing the micelles in the presence vs. absence of triglyceride. Dark cyan line represents the fitted micellar region $\left(Q>0.02 \AA^{-1}\right)$. Schematic on the right shows the dimensions generated from the fitting results being similar in both cases. In addition, it depicts the lack of interaction between the micelles and the lipid droplets. Error bars for the intensity scattering data are smaller than the symbol size.

Figure 8 overlays the scattering plots of the samples containing model bile micelles in the presence vs. absence of triglyceride. The intensity plots fully overlap in the medium to high $\mathrm{Q}$ range $\left(0.01 \AA^{-1}<\mathrm{Q}<0.2 \AA^{-1}\right)$, the region corresponding to the bile micelles, suggesting no change in the structure of bile micelles in the presence of the triglyceride, as reflected in the fitting results (Figure 8, right). The concentration of micelles in the presence of lipid determined by the fit was $1.30 \times 10+17$ micelles/mL, which is within the error of the micelle concentration determined in the absence of lipid $(9.1 \times 10+16 \pm 1.2 \times 10+16$ micelles/mL), indicating negligible change in micellar concentration in the presence of lipids. As these results are counterintuitive considering the widely accepted impact of bile in mediating the lipid emulsification process in the intestine, the experiment was repeated using various triolein concentrations (Figure 9). 


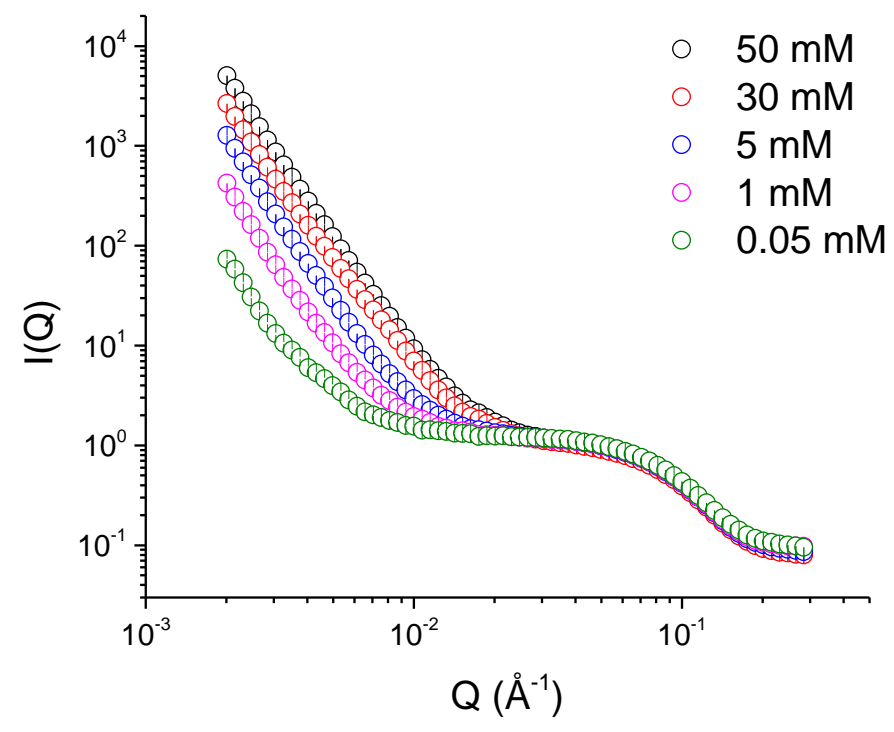

Figure 9: Intensity scattering plots of triolein mixed with fed state bile micelles at various triolein concentrations. Error bars for the intensity scattering data are smaller than the symbol size. Fits are not shown to avoid cluttering. Fitting results are summarized in Table 5.

Table 5: Spheroid shape model fitting results for samples containing various concentrations of triolein mixed with bile micelles at fed concentrations

\begin{tabular}{|c|c|c|c|c|c|c|}
\hline $\begin{array}{c}\text { Triolein } \\
\text { Conc. }(\mathbf{m M})\end{array}$ & Background & $\begin{array}{c}\text { Major } \\
\text { radius } \mathbf{( n m )}\end{array}$ & $\begin{array}{c}\text { Minor } \\
\text { radius (nm) }\end{array}$ & $\begin{array}{c}\text { Polydispersity } \\
\text { in minor } \\
\text { radius }\end{array}$ & $\begin{array}{c}\text { Scale } \\
\text { (mL/mL } \\
\text { solution) }\end{array}$ & $\begin{array}{c}\text { Micelle } \\
\text { concentration } \\
\text { (\#/mL) }\end{array}$ \\
\hline $50 \mathrm{mM}$ & 0.0865 & 2.9 & 1.8 & 0.22 & 0.00520 & $1.30 \times 10+17$ \\
\hline $30 \mathrm{mM}$ & 0.0781 & 3.0 & 1.7 & 0.25 & 0.00518 & $1.41 \times 10+17$ \\
\hline $5 \mathrm{mM}$ & 0.0843 & 3.0 & 1.7 & 0.27 & 0.00554 & $1.52 \times 10+17$ \\
\hline $1 \mathrm{mM}$ & 0.0938 & 3.0 & 1.8 & 0.22 & 0.00527 & $1.25 \times 10+17$ \\
\hline $0.05 \mathrm{mM}$ & 0.0936 & 3.0 & 1.7 & 0.27 & 0.00566 & $1.57 \times 10+17$ \\
\hline $0 \mathrm{mM}^{*}$ & 0.0872 & 3.5 & 1.9 & 0 & 0.00444 & $8.02 \times 10+16$ \\
\hline
\end{tabular}

* Fitting result of the scattering profile of micelle solutions prepared on Dec 2011 (same as Table 4).

The bile micelle regions $\left(0.01 \AA^{-1}<\mathrm{Q}<0.2 \AA^{-1}\right)$ of the intensity scattering plots overlap for all the TG concentrations tested. This observation and the fitting results (Table 5) confirm that bile micelles are not affected by the presence of TG in solution. Bile salts have shown high adsorption affinity for lipids but do not reach high oil-water interface pressures, suggesting a low bile salt concentration adsorbing to the surface compared to more standard surfactants such as SDS and Tween 20 [46]. A study by Tiss et al. showed that NaTDC, the type of BS used in this study, forms a compact interfacial monolayer at the surface of oil droplets. The BS adsorbs rapidly upon mixing with a constant interfacial density even at solute NaTDC concentrations 
below the critical micellar concentration [55]. This observation is thought to be due to the flat planar structure of bile salts $[46,47]$. The interfacial area of a NaTDC molecule at the oil-water interface was found to be $115 \AA^{2}$ [55]. For the system studied here, containing fed state simulated intestinal fluids mixed with $50 \mathrm{mM}$ triolein, the concentration of NaTDC molecules adsorbed to the surface of oil droplets, assuming a tightly packed interfacial surface, can range between $0.04-0.8 \mu \mathrm{M}$ depending on the mean size of oil droplets (range used: $0.5-10 \mu \mathrm{m}$ diameter). This portion of BS partitioned into oil is insignificant compared to the total amount of BS present $(12 \mathrm{mM})$ in solution, thus justifying the absence of effect of oil droplets on the structure of bile micelles.

Enzymatic cocktail (Experiment 3b) - The scattering plots for samples containing bile micelles in the presence vs. absence of pancreatin extract were overlayed (Figure 10) to determine whether physical (e.g., hydrophobic) interactions occur between micelles and components of the pancreatin extract. Enzymatic activity was inhibited as soon as pancreatin extract was mixed with the micellar solution to mimic the inhibitor-containing lipolysis samples used in Experiment 2. Pancreatin extract generated some scattering intensity in the low Q region $\left(\mathrm{Q}<0.01 \AA^{-1} \rightarrow 50-200 \mathrm{~nm}\right.$ diameter range, roughly). This suggests the presence of large proteins or protein aggregates in the extract. The scattering plots overlap in the medium to high Q range $\left(0.01 \AA^{-1}<\mathrm{Q}<0.2 \AA^{-1}\right)$ indicating the mixture of pancreatin, inhibitor, and methanol have no effect on the structure and number of bile micelles. This result is important, as it alleviates concern that methanol, used as a solvent for inhibitor in these experiments, may impact micelle structures. 


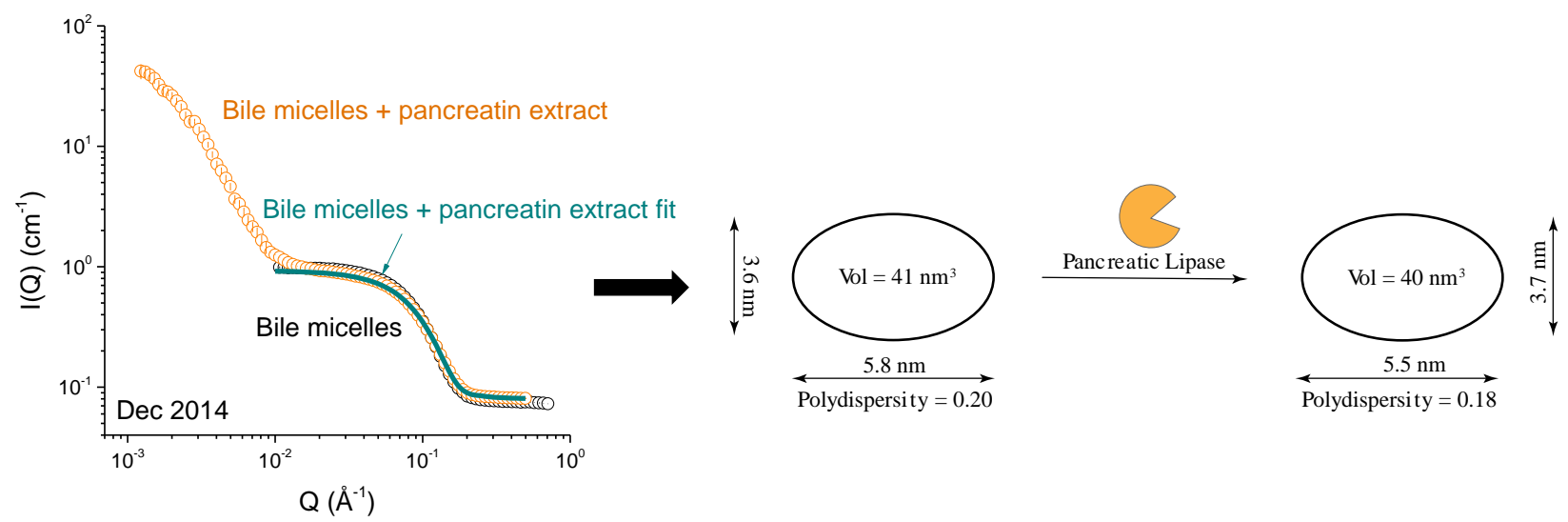

Figure 10: Scattering intensity plot comparison of the micelles in the presence vs. absence of enzymatic cocktail. Dark cyan line represents the fitted micellar region $\left(Q>0.02 \AA^{-1}\right)$. Schematic on the right shows the dimensions obtained from fitting the two graphs. The similar fitting results suggest no structural change and a lack of physical interaction between the micelles and the pancreatin extract. Error bars for the intensity scattering data are smaller than the symbol size.

\section{Diglyceride (Experiment 3c) - A few studies have shown, for example by} ultracentrifugation of human intestinal content during the in vivo digestion of corn oil, that diglycerides do not partition into bile micelles $[7,56]$. Instead, they remain at the surface of oil droplets until they are further digested into monoglycerides and fatty acids. The goal of comparing scattering intensity plots in the presence vs. absence of DG (Figure 11) was to validate this phenomenon for our in vitro system.

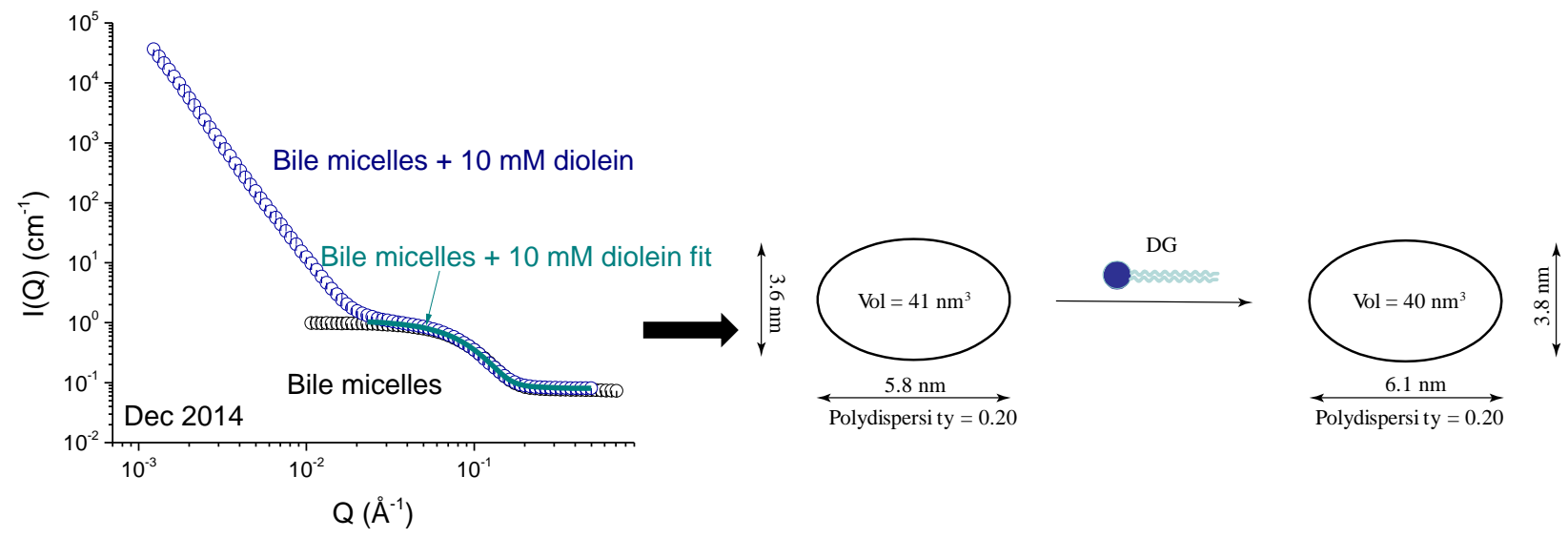

Figure 11: Scattering intensity plot of simulated bile micelles in the presence vs. absence of diglycerides (DG). Dark cyan line represents the fitted micellar region $\left(Q>0.02 \AA^{-1}\right)$. The low $Q$ range $\left(Q<0.01 \AA^{-1}\right)$ suggests the presence of larger particles formed by the presence of DG in solution. The medium to high $Q$ range $\left(0.01 \AA^{-1}<Q<0.2 \AA^{-1}\right)$ shows overlapping scattering intensities, indicating the micellar size not being affected by the DG in solution, reflected in the similar fitting results (right) and suggesting a lack of interaction between the DG and the micelles. Error bars for the intensity scattering data are smaller than the symbol size. 
The scattering plots overlapping in the medium to high $Q$ region $\left(0.01 \AA^{-1}<Q<0.2 \AA^{-1}\right)$ indicate similar shape and size between the two samples, supported by the fitting results. As the DG molecules are unable to insert themselves into the bile micelles, they may form larger structures such as lipid droplets or lipid bilayers. The slope in the low $Q\left(Q<0.01 \AA^{-1}\right)$ portion of the scattering intensity graph for the preparation containing DG suggests the presence of these larger structures.

Monoglyceride (Experiment 3d) - Along with fatty acids, monoglycerides are products of lipolysis known to affect the size of micelles. Studies support the elongation of micelles at increasing MG concentrations $[11,12]$. The scattering intensity results from our experiment are shown in Figure 12.

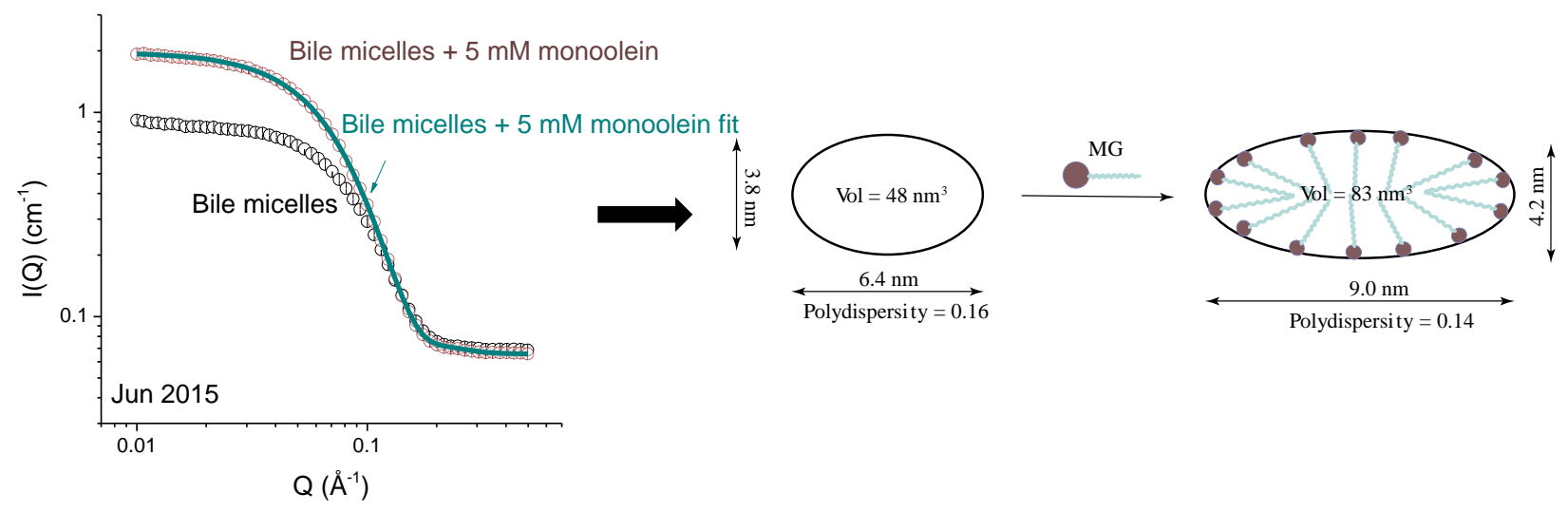

Figure 12: Scattering intensity plot of micelles in the presence vs. absence of monoglyceride (MG). Dark cyan line represents the fitted micellar region $\left(Q>0.02 \AA^{-1}\right)$. Fitting results are shown on the right. $A 120 \%$ increase in micellar volume occurs in the presence of $5 \mathrm{mM}$ MG. Error bars for the intensity scattering data are smaller than the symbol size.

Figure 12 shows the scattering intensity plots of the model bile micelles in the presence vs. absence of MG. MG significantly influences the size of micelles by inserting into the micelle, causing elongation along the major axis and volume increase. This elongation phenomenon is consistent with the behavior of micelles observed during in vitro lipolysis (section 3.2).

Fatty acid (Experiment 3e) - Fatty acids are also known to affect the size of bile micelles by partitioning and causing micelles to elongate along one dimension $[12,51]$. Similar to MG, 
the FA cause the micelles to swell (Figure 13) consistent with the behavior observed during in vitro lipolysis (section 3.2).

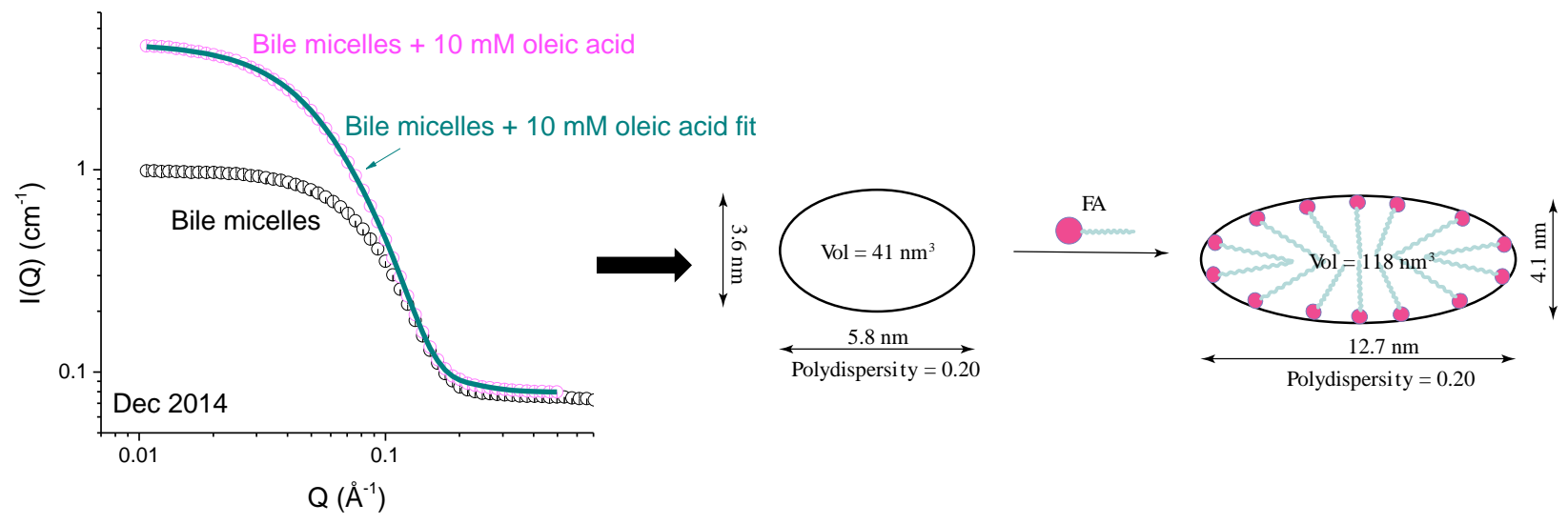

Figure 13: Scattering intensity plot of bile micelles in the presence vs. absence of fatty acid (FA). Dark cyan line represents the fitted micellar region $\left(Q>0.02 \AA^{-1}\right)$. The significant difference in the size of micelles is supported by fitting results (right). A $198 \%$ increase in micellar volume occurs in the presence of $10 \mathrm{mM}$ FA. Error bars for the intensity scattering data are smaller than the symbol size.

In summary, these results show that mixtures of bile micelles with oil droplets, pancreatin extract, and DG separately caused no change in micelle size and shape. Hence, it was concluded that micelle interactions with these components are negligible in our digestion model. For mixtures of bile micelles with FA and MG, obvious micellar swelling occurs as observed during in vitro lipolysis. This agrees with literature reports on the effect of FA and MG on the size of bile micelles, due to the micelles serving as a niche for amphiphilic compound partitioning [11, $12,51]$

This study has laid the groundwork in describing in a mechanistic and quantitative fashion the evolution of bile micelles during the intestinal digestion of lipids. A thorough study of the concentration effect of FA and MG on the volume of micelles could allow for a quantitative approach to describing and predicting the effect of lipids and intestinal lipolysis kinetics on the micellar volume. These results can ultimately be used to predict drug partitioning as dynamic changes in colloidal structures occur due to the presence of lipids in the intestine. 
Coupled with drug dissolution and absorption models, this information could help enable quantitative prediction of the effect of ingested lipids on the absorption of these compounds.

\section{Conclusions}

Lipids have a marked ability to enhance drug solubility in the intestine [1, 3, 57-61]. In particular, dissolution rate-limited poorly water-soluble drugs can experience enhanced solubility and ultimately absorption in the presence of lipids due to increased partitioning into the colloidal structures that are evolving in the intestine during lipolysis. The increased drug concentration in the intestinal lumen serves as a driving force for absorption, resulting in enhanced drug bioavailability. The impact of lipids on overall drug absorption is, however, not amenable to quantitative prediction, in part due to incompletely defined physicochemical changes of colloids in the intestinal lumen. Bile micelles are one of the main colloidal species in the intestine known to serve as a compound carrier along the GI tract and into the intestinal mucosa $[2,62]$. Their role as carriers is enhanced in the presence of lipids [5, 63]. As a result, understanding the changes in their physicochemical properties during lipid digestion is critical in predicting and exploiting their effects in compound delivery in the GI tract [3]. The structure of bile micelles before and during an in vitro intestinal digestion process, as well as the impact of each of the molecular components present during digestion on the bile micelle structure, were studied using SANS. We demonstrated that the evolution of micelle structure can be monitored in real time during the digestion of a lipid as a model food source. The micelles were concluded to be elongating as digestion proceeded causing an increase in micellar volume. The elongation process was due to the insertion of FA and MG, two of the lipolysis products, within the micelles. The kinetics of volume change during digestion were non-linear and similar to those of TG lipolysis, suggesting the ability to quantitatively predict micellar volume based on lipolysis 
kinetics and vice-versa. The quantitative change in micelle volume measured as a function of extent of digestion and concentrations of digestion components can aid in developing predictive models of the overall impact of lipids on drug compound absorption. Ultimately, these models can be exploited to design better lipid-based oral drug delivery methods.

\section{Acknowledgements}

This study was supported by the National Institutes of Health grant no. NIGMS R01EB013037. We would like to acknowledge Yun Liu from the National Institute of Standards and Technology (NIST) Center for Neutron Research for his guidance with our experimental work and data analysis. We would also like to thank Yan Yan Yeap for her help with sample preparation.

\section{References}

1. Di Maio, S. and R.L. Carrier, Gastrointestinal contents in fasted state and post-lipid ingestion: in vivo measurements and in vitro models for studying oral drug delivery. J Control Release, 2011. 151(2): p. 110-22.

2. Holm, R., A. Müllertz, and H. Mu, Bile salts and their importance for drug absorption. International Journal of Pharmaceutics, 2013. 453(1): p. 44-55.

3. Porter, C.J., N.L. Trevaskis, and W.N. Charman, Lipids and lipid-based formulations: optimizing the oral delivery of lipophilic drugs. Nat Rev Drug Discov, 2007. 6(3): p. 231-48.

4. Phan Ct Fau - Tso, P. and P. Tso, Intestinal lipid absorption and transport. Front Biosci, 2001. 6: p. D299-319.

5. Rice, S.A., Small angle scattering of X-rays. A. Guinier and G. Fournet. Translated by C. B. Wilson and with a bibliographical appendix by K. L. Yudowitch. Wiley, New York, 1955. 268 pp. \$7.50. Journal of Polymer Science, 1956. 19(93): p. 594-594.

6. Kiselev, M.A., et al., Structural transition in aqueous lipid/bile salt [DPPC/NaDC] supramolecular aggregates: SANS and DLS study. Chemical Physics, 2013. 424: p. 93-99.

7. Hjelm, R.P., et al., Form and structure of self-assembling particles in monoolein-bile salt mixtures. The Journal of Physical Chemistry, 1995. 99(44): p. 16395-16406.

8. Mazer, N.A., G.B. Benedek, and M.C. Carey, Quasielastic light-scattering studies of aqueous biliary lipid systems. Mixed micelle formation in bile salt-lecithin solutions. Biochemistry, 1980. 19(4): p. 601-615.

9. Sek, L., et al., Evaluation of the in-vitro digestion profiles of long and medium chain glycerides and the phase behaviour of their lipolytic products. J Pharm Pharmacol, 2002. 54(1): p. 29-41.

10. Lin, T.L., et al., The use of small-angle neutron scattering to determine the structure and interaction of dihexanoylphosphatidylcholine micelles. Journal of the American Chemical Society, 1986. 108(12): p. 3499-3507. 
11. Kossena, G.A., et al., Probing drug solubilization patterns in the gastrointestinal tract after administration of lipid-based delivery systems: A phase diagram approach. Journal of Pharmaceutical Sciences, 2004. 93(2): p. 332-348.

12. Hjelm, R.P., et al., Structure of Conjugated Bile Salt-Fatty Acid-Monoglyceride Mixed Colloids: Studies by Small-Angle Neutron Scattering. The Journal of Physical Chemistry B, 2000. 104(2): p. 197-211.

13. Garidel, P., et al., Membranolytic Activity of Bile Salts: Influence of Biological Membrane Properties and Composition. Molecules, 2007. 12(10): p. 2292.

14. Andre Guinier, G.F., Small-Angle Scattering of X-Rays. 1955, New York: John Wiley and Sons, Inc.

15. Madenci, D., et al., Simple model for the growth behaviour of mixed lecithin-bile salt micelles. Phys Chem Chem Phys, 2011. 13(8): p. 3171-8.

16. Vors, C., et al., Coupling in vitro gastrointestinal lipolysis and Caco-2 cell cultures for testing the absorption of different food emulsions. Food Funct, 2012. 3(5): p. 537-46.

17. Birru, W.A., et al., Digestion of Phospholipids after Secretion of Bile into the Duodenum Changes the Phase Behavior of Bile Components. Molecular Pharmaceutics, 2014. 11(8): p. 2825-2834.

18. Carriere, F., et al., Quantitative study of digestive enzyme secretion and gastrointestinal lipolysis in chronic pancreatitis. Clin Gastroenterol Hepatol, 2005. 3(1): p. 28-38.

19. Egelhaaf, S.U., J.S. Pedersen, and P. Schurtenberger, Shape transformations in biological mixed surfactant systems: from spheres to cylinders to vesicles, in Trends in Colloid and Interface Science IX, J. Appell and G. Porte, Editors. 1995, Steinkopff. p. 224-227.

20. Phan, S., et al., Disposition and crystallization of saturated fatty acid in mixed micelles of relevance to lipid digestion. Journal of Colloid and Interface Science, 2015. 449(0): p. 160-166.

21. Fatouros, D.G., B. Bergenstahl, and A. Mullertz, Morphological observations on a lipid-based drug delivery system during in vitro digestion. European Journal of Pharmaceutical Sciences, 2007. 31(2): p. 85-94.

22. Kossena, G.A., et al., Separation and characterization of the colloidal phases produced on digestion of common formulation lipids and assessment of their impact on the apparent solubility of selected poorly water-soluble drugs. J Pharm Sci, 2003. 92(3): p. 634-48.

23. Phan, S., et al., Structural aspects of digestion of medium chain triglycerides studied in real time using SSAXS and Cryo-TEM. Pharm Res, 2013. 30(12): p. 3088-100.

24. Warren, D.B., et al., Real time evolution of liquid crystalline nanostructure during the digestion of formulation lipids using synchrotron small-angle X-ray scattering. Langmuir, 2011. 27(15): p. 9528-34.

25. Salentinig, S., et al., Transitions in the internal structure of lipid droplets during fat digestion. Soft Matter, 2011. 7(2): p. 650-661.

26. Phan, S., et al., How relevant are assembled equilibrium samples in understanding structure formation during lipid digestion? Eur J Pharm Biopharm, 2015. 96: p. 117-24.

27. Kline, S., Reduction and analysis of SANS and USANS data using IGOR Pro. Journal of Applied Crystallography, 2006. 39(6): p. 895-900.

28. DANSE. SasView for Small Angle Scattering Analysis, NSF project DMR-0520547. 2012; Available from: www.sasview.org.

29. Buyukozturk, F., et al., Effect of ingested lipids on drug dissolution and release with concurrent digestion: a modeling approach. Pharm Res, 2013. 30(12): p. 3131-44.

30. Sek, L., C.J. Porter, and W.N. Charman, Characterisation and quantification of medium chain and long chain triglycerides and their in vitro digestion products, by HPTLC coupled with in situ densitometric analysis. J Pharm Biomed Anal, 2001. 25(3-4): p. 651-61.

31. Small, D.M., M.C. Bourges, and D.G. Dervichian, The biophysics of lipidic associations. I. The ternary systems: lecithin-bile salt-water. Biochim Biophys Acta, 1966. 125(3): p. 563-80. 
32. Fernandez, S., et al., Lipolysis of the semi-solid self-emulsifying excipient Gelucire ${ }^{\circledR} 44 / 14$ by digestive lipases. Biochimica et Biophysica Acta (BBA) - Molecular and Cell Biology of Lipids, 2008. 1781(8): p. 367-375.

33. Kanicky, J.R. and D.O. Shah, Effect of degree, type, and position of unsaturation on the pKa of long-chain fatty acids. J Colloid Interface Sci, 2002. 256(1): p. 201-7.

34. Salentinig, S., L. Sagalowicz, and O. Glatter, Self-assembled structures and pKa value of oleic acid in systems of biological relevance. Langmuir, 2010. 26(14): p. 11670-9.

35. Cistola, D.P., et al., Ionization and phase behavior of fatty acids in water: application of the Gibbs phase rule. Biochemistry, 1988. 27(6): p. 1881-8.

36. Olsson, P., J. Holmback, and B. Herslof, A single step reversed-phase high performance liquid chromatography separation of polar and non-polar lipids. J Chromatogr A, 2014. 1369: p. 10515.

37. Müllertz, A., et al., Insights into Intermediate Phases of Human Intestinal Fluids Visualized by Atomic Force Microscopy and Cryo-Transmission Electron Microscopy ex Vivo. Molecular Pharmaceutics, 2012. 9(2): p. 237-247.

38. Riethorst, D., et al., An In-Depth View into Human Intestinal Fluid Colloids: Intersubject Variability in Relation to Composition. Molecular Pharmaceutics, 2016. 13(10): p. 3484-3493.

39. Hjelm, R.P., P. Thiyagarajan, and H. Alkan-Onyuksel, Organization of phosphatidylcholine and bile salt in rodlike mixed micelles. The Journal of Physical Chemistry, 1992. 96(21): p. 8653-8661.

40. Bacon, G., Neutron Diffraction. 3rd ed. 1980, New York: Oxford University Press.

41. Hjelm, R.P., Jnr, P. Thiyagarajan, and H. Alkan, A small-angle neutron scattering study of the effects of dilution on particle morphology in mixtures of glycocholate and lecithin. Journal of Applied Crystallography, 1988. 21(6): p. 858-863.

42. Kaya, H., Scattering from cylinders with globular end-caps. Journal of Applied Crystallography, 2004. 37(2): p. 223-230.

43. Kaya, H. and N.-R. de Souza, Scattering from capped cylinders. Addendum. Journal of Applied Crystallography, 2004. 37(3): p. 508-509.

44. Fink, H.P., Structure analysis by small-angle X-ray and neutron scattering. Von L. A. FEIGIN und D. I. SVERGUN. ISBN 0-306-42629-3. New York/London: Plenum Press 1987. XIII, 335 S., geb. US \$ 95.40. Acta Polymerica, 1989. 40(3): p. 224-224.

45. Kotlarchyk, M., R.B. Stephens, and J.S. Huang, Study of Schultz distribution to model polydispersity of microemulsion droplets. The Journal of Physical Chemistry, 1988. 92(6): p. 1533-1538.

46. Maldonado-Valderrama, J., et al., The role of bile salts in digestion. Adv Colloid Interface Sci, 2011. 165(1): p. 36-46.

47. Golding, M. and T.J. Wooster, The influence of emulsion structure and stability on lipid digestion. Current Opinion in Colloid \& Interface Science, 2010. 15(1-2): p. 90-101.

48. Long, M.A., et al., Characterization of Lecithin-Taurodeoxycholate Mixed Micelles Using SmallAngle Neutron Scattering and Static and Dynamic Light Scattering. The Journal of Physical Chemistry, 1994. 98(16): p. 4402-4410.

49. Charlton, I.D. and A.P. Doherty, Electrolyte-Induced Structural Evolution of Triton X-100 Micelles. The Journal of Physical Chemistry B, 2000. 104(34): p. 8327-8332.

50. Dungan, S.R., B.H. Tai, and N.I. Gerhardt, Transport mechanisms in the micellar solubilization of alkanes in oil-in-water emulsions. Colloids and Surfaces A: Physicochemical and Engineering Aspects, 2003. 216(1-3): p. 149-166.

51. Kossena, G.A., et al., Influence of the intermediate digestion phases of common formulation lipids on the absorption of a poorly water-soluble drug. J Pharm Sci, 2005. 94(3): p. 481-92. 
52. Persson, E.M., et al., A Clinical Single-Pass Perfusion Investigation of the Dynamic in Vivo Secretory Response to a Dietary Meal in Human Proximal Small Intestine. Pharmaceutical Research, 2006. 23(4): p. 742-751.

53. Kohli, K., et al., Self-emulsifying drug delivery systems: an approach to enhance oral bioavailability. Drug Discovery Today, 2010. 15(21-22): p. 958-965.

54. Kalepu, S., M. Manthina, and V. Padavala, Oral lipid-based drug delivery systems - an overview. Acta Pharmaceutica Sinica B, 2013. 3(6): p. 361-372.

55. Tiss, A., et al., Surface behaviour of bile salts and tetrahydrolipstatin at air/water and oil/water interfaces. Chem Phys Lipids, 2001. 111(1): p. 73-85.

56. Garner, C.W., Boronic acid inhibitors of porcine pancreatic lipase. J Biol Chem, 1980. 255(11): p. 5064-8.

57. Singh, B.N., Effects of food on clinical pharmacokinetics. Clin Pharmacokinet, 1999. 37(3): p. 21355.

58. Singh, B., et al., Recent advances in self-emulsifying drug delivery systems (SEDDS). Crit Rev Ther Drug Carrier Syst, 2014. 31(2): p. 121-85.

59. Mullertz, A., et al., New perspectives on lipid and surfactant based drug delivery systems for oral delivery of poorly soluble drugs. J Pharm Pharmacol, 2010. 62(11): p. 1622-36.

60. Rangel-Yagui, C.O., A. Pessoa, Jr., and L.C. Tavares, Micellar solubilization of drugs. J Pharm Pharm Sci, 2005. 8(2): p. 147-65.

61. O'Driscoll, C.M. and B.T. Griffin, Biopharmaceutical challenges associated with drugs with low aqueous solubility--the potential impact of lipid-based formulations. Adv Drug Deliv Rev, 2008. 60(6): p. 617-24.

62. Griffin, B.T., et al., Comparison of in vitro tests at various levels of complexity for the prediction of in vivo performance of lipid-based formulations: Case studies with fenofibrate. European Journal of Pharmaceutics and Biopharmaceutics, 2014. 86(3): p. 427-437.

63. Bhattacharjee, J., et al., Tween 80-Sodium Deoxycholate Mixed Micelles: Structural Characterization and Application in Doxorubicin Delivery. The Journal of Physical Chemistry B, 2010. 114(49): p. 16414-16421. 